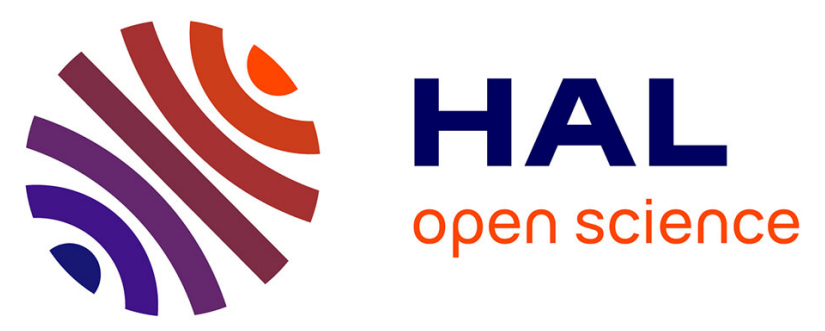

\title{
A multiscale LATIN/FAS algorithm with time-space model reduction for frictional contact problems
}

Anthony Giacoma, David Dureisseix, Anthony Gravouil, Michel Rochette

\section{To cite this version:}

Anthony Giacoma, David Dureisseix, Anthony Gravouil, Michel Rochette. A multiscale LATIN/FAS algorithm with time-space model reduction for frictional contact problems. International Journal for Numerical Methods in Engineering, 2014, 97 (3), pp.207-230. 10.1002/nme.4590 . hal-00921821

\section{HAL Id: hal-00921821 \\ https://hal.science/hal-00921821}

Submitted on 19 Jan 2016

HAL is a multi-disciplinary open access archive for the deposit and dissemination of scientific research documents, whether they are published or not. The documents may come from teaching and research institutions in France or abroad, or from public or private research centers.
L'archive ouverte pluridisciplinaire HAL, est destinée au dépôt et à la diffusion de documents scientifiques de niveau recherche, publiés ou non, émanant des établissements d'enseignement et de recherche français ou étrangers, des laboratoires publics ou privés. 


\title{
A multiscale LATIN/FAS algorithm with time-space model reduction for frictional contact problems
}

\author{
A. Giacoma ${ }^{1,2}$, D. Dureisseix ${ }^{* 1}$, A. Gravouil ${ }^{1,3}$, and M. Rochette ${ }^{2}$ \\ ${ }^{1}$ Université de Lyon, INSA-Lyon, LaMCoS, CNRS UMR 5259, \\ F-69621, Villeurbanne (France) \\ ${ }^{2}$ ANSYS France, Villeurbanne (France) \\ ${ }^{3}$ Institut universitaire de France
}

\begin{abstract}
A multiscale strategy using model reduction for frictional contact computation is presented. This new approach aims to improve computation time of finite element simulations involving frictional contact between linear and elastic bodies. This strategy is based on a combination between the LATIN (LArge Time INcrement) method and the FAS (Full Approximation Scheme) multigrid solver. The LATIN method is an iterative solver operating on the whole time-space domain. Applying an a posteriori analysis on solutions of different frictional contact problems shows a great potential as far as reducibility for frictional contact problems is concerned. Time-space vectors forming the so-called reduced basis depict particular scales of the problem. It becomes easy to make analogies with multigrid method to take full advantage of multiscale information.

This is a preprint of an article published in its final form in International Journal for Numerical Methods in Engineering 97(3):207-230, 2014, Wiley. doi:10.1002/nme.4590
\end{abstract}

Keywords: Frictional contact, LATIN, Model reduction, Proper Orthogonal Decomposition (POD), Multigrid (MG-FAS).

\section{Introduction}

Simulations of mechanical systems involving frictional contact between flexible bodies can lead to strong difficulties. Non-linear behavior for bodies, large contact zones, and somehow large friction coefficient lead to numerical issues. Frictional contact problems are characterized by non-smooth behaviors at contacting boundaries of solids (discontinuities at contact interfaces for both normal

${ }^{*}$ LaMCoS (Laboratoire de Mécanique des Contacts et des Structures), Bat. J. d'Alembert, 18 rue des Sciences, F-69621 Villeurbanne Cedex (France). E-Mail: david.dureisseix@insalyon.fr. 
and tangential components). These strong non-linearities dare the computational mechanic community to solve efficiently such problems. Over the last decades, various resolution methods have been developed. Contact problems can be formulated as an optimization problem subject to constraints with the augmented Lagrangian formulation. Such formulation can be solved using augmented Lagrangian methods $[1,2,3,4,5,6,7,8]$. Non-linear Gauss-Seidel solvers are also developed and applied to problems involving multi-contact bodies (rigid or flexible) $[9,10,11]$. But these last methods are not the most efficient to solve large systems (poor rate of convergence). Gradient methods are developed and adapted $[12,13,14,15]$ (with projection of the descent direction on feasible region for contact laws or active-set violation control strategy ...) in order to reproduce well-known performances of such methods for linear systems. But the main drawback of them is the difficulty to provide a convergence proof.

In spite of the non-smooth property of contact problems, widely used Newton's methods (generalized Newton's methods) are studied [16, 17, 18, 19]. Such methods proved their efficiency and robustness in large scale elasticity problems with contact and friction. Once again and due non-smooth property of contact problems, convergence proof of generalized Newton's methods are hard to provide. Another method consists in casting the contact problems into a linear complementarity problem (LCP) and using specific solvers such as active-set methods, Lemke's algorithm, projected successive over relaxation (PSOR). In [20], an LCP formulation is used to solve a frictional contact problem by faceting the Coulomb's cone. Nevertheless, this approximation is tough and not efficient (the problem to solve becomes larger). Nowadays, LCP formulations are suited to frictionless problems.

Generally speaking and even within a quasi-static context, all these nonlinear solvers can lead to prohibitive time of computations. Acceleration strategies based on multigrid methods were proposed [21, 22]. Computational costs spur recent and intensive works on efficient model reduction techniques in various fields [23, 24, 25, 26]. But and due to the non-differentiable nature of frictional contact, application of such methods seems to be heretic.

The aim of this work is to propose a strategy accelerating solution for frictional contact problems in the finite element framework embedding model reduction techniques also well-suited for parametric studies. Linear elastic, homogeneous, isotropic behavior and quasi-static evolution are assumed for the bodies. Frictional contact laws involve a non-linear and non-smooth behavior at the boundary of the body. To solve this mechanical problem, the nonincremental LATIN (LArge Time INcrement) method is used. This method is well-known for its ability to solve difficult non-linear large problems (nonlinear material, contact problems...) [27, 28] with a global time-space approach. This method is closed to augmented Lagrangian methods. Its great advantages are non re-factorization of matrices (stiffness matrix remains constant through LATIN iterations) and explicit subsequent iterations (no iterations to handle the non-linear behavior solved only on the contacting boundary). So the cost of a LATIN iteration is low even if the number of iterations can be elevated as augmented Lagrangian methods. We will try to exploit scale separability of contact problems as observed in $[29,30]$ for fracture mechanics. The set of vector solutions can be compressed into a small number of vectors. Moreover, each of them describes a particular scale of the problem (e.g. scale of the global structure $v s$. scale of the crack). This phenomenon fosters multiscale strategies. 


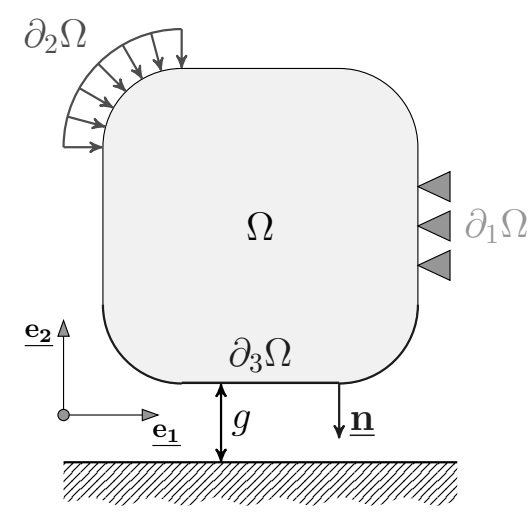

Figure 1: Problem set.

At first, we propose to illustrate multiscale contents of solutions for three different contact problems. Then, some recalls about the LATIN method will be given in order to introduce the specific approach we propose. Subsequently, applications with convergence results on those three cases will be performed in order to highlight the feasibility of our strategy and its aptitude in the parametric studies framework.

\section{Reference problem}

A flexible body is contacting a rigid basement (figure 1). We define $\Omega$, the domain occupied by this solid with an open boundary (denoted by $\partial \Omega$ ) at time $t \in[0, T] \subset \mathbb{R}$. This boundary can be split into three parts:

- $\partial_{1} \Omega$ : the part of the boundary where displacements $\underline{\mathbf{u}}_{\mathrm{p}}$ are prescribed;

- $\partial_{2} \Omega=\partial \Omega \backslash \partial_{1} \Omega$ : the part of the boundary where external loads $\underline{\mathbf{f}}_{\text {ext }}$ are prescribed;

- $\partial_{3} \Omega$ : the part of the boundary where contact conditions occurs (contacting interface).

\subsection{Strong formulation}

Linear hypothesis are assumed (small displacements, homogeneous-isotropicelastic material). External loads are time dependent but quasi-static regime is assumed. The problem can be set as follows: find the displacement field and the Cauchy's stress field denoted respectively by $\underline{\mathbf{u}}(\underline{\mathbf{x}}, t)$ and $\boldsymbol{\sigma}(\underline{\mathbf{x}}, t)$ satisfying:

- kinematic admissibility:

$$
\underline{\mathbf{u}}(\underline{\mathbf{x}}, t) \in \mathcal{U}^{[0, T]}
$$

with $\mathcal{U}^{[0, T]}=\left\{\underline{\mathbf{u}}\right.$ has a finite energy, $\left.\forall(\underline{\mathbf{x}}, t) \in \partial_{1} \Omega \times[0, T], \underline{\mathbf{u}}(\underline{\mathbf{x}}, t)=\underline{\mathbf{u}}_{\mathrm{p}}\right\}$ 
and the strain-displacement (in small perturbations) relationship reads:

$$
\forall(\underline{\mathbf{x}}, t) \in \Omega \times[0, T]: \quad \varepsilon(\underline{\mathbf{u}})=\frac{1}{2}\left[\boldsymbol{\nabla} \underline{\mathbf{u}}+\nabla^{T} \underline{\mathbf{u}}\right]
$$

- static admissibility: $\boldsymbol{\sigma}(\underline{\mathbf{x}}, t)$ is balanced with external force, i.e.

$$
\begin{cases}\forall(\underline{\mathbf{x}}, t) \in \partial_{2} \Omega \times[0, T]: & \boldsymbol{\sigma} \underline{\mathbf{n}}=\underline{\mathbf{f}}_{\mathrm{ext}} \\ \forall(\underline{\mathbf{x}}, t) \in \Omega \times[0, T]: & \underline{\operatorname{div}}(\boldsymbol{\sigma})=\underline{\mathbf{0}}\end{cases}
$$

- constitutive laws:

- Hooke law for elasticity :

$$
\forall(\underline{\mathbf{x}}, t) \in \Omega \times[0, T]: \quad \boldsymbol{\sigma}=\mathbb{K} \varepsilon(\underline{\mathbf{u}})
$$

where $\mathbb{K}$ is the linear elasticity operator.

- Coulomb frictional law for contact. With $\underline{\mathbf{v}}$ and $\underline{\boldsymbol{\lambda}}$, the traces of displacement field and contact force field (both normal and tangential) on the contacting interface $\partial_{3} \Omega$, these conditions can be written formally as:

$$
\mathcal{R}(\underline{\mathbf{v}}, \underline{\boldsymbol{\lambda}})=0
$$

To be more precise for the frictional behavior, at the contacting interface $\partial_{3} \Omega$, we denote the local outward normal vector $\underline{\mathbf{n}}$. Then, the two fields can be split into a normal and a tangential part as:

$$
\forall(\underline{\mathbf{x}}, t) \in \partial_{3} \Omega \times[0, T]: \quad\left\{\begin{array}{l}
\underline{\mathbf{v}}(\underline{\mathbf{x}}, t)=u_{N} \underline{\mathbf{n}}+\underline{\mathbf{u}}_{T} \\
\underline{\boldsymbol{\lambda}}(\underline{\mathbf{x}}, t)=\lambda_{N} \underline{\mathbf{n}}+\underline{\boldsymbol{\lambda}}_{T}
\end{array}\right.
$$

$\mathcal{R}$ governs the contact behavior at the interface $\forall(\underline{\mathbf{x}}, t) \in \partial_{3} \Omega \times[0, T]$, according to Signorini's conditions and Coulomb's law on the displacement field and contact force field:

- Normal contact or Signorini's conditions given in [31],

$$
\begin{aligned}
u_{N}=\underline{\mathbf{u}} \cdot \underline{\mathbf{n}} \leqslant g & \text { Non-penetration condition } \\
\lambda_{N}=(\boldsymbol{\sigma} \underline{\mathbf{n}}) \cdot \underline{\mathbf{n}} \leqslant 0 & \text { Compressive contact force } \\
\lambda_{N}\left(g-u_{N}\right)=0 & \text { Complementarity condition }
\end{aligned}
$$

where $g$ is the initial gap.

- Tangential contact or Coulomb's friction law [32]:

$$
\begin{array}{ll}
\left\|\underline{\boldsymbol{\lambda}}_{T}\right\|_{2}=-\mu \lambda_{N} & \text { sliding: }\left\|\underline{\dot{\mathbf{u}}}_{T}\right\|_{2} \neq 0 \text { and } \underline{\dot{\mathbf{u}}}_{T}=-\rho \underline{\boldsymbol{\lambda}}_{T} \text { with } \rho>0 \\
\left\|\underline{\boldsymbol{\lambda}}_{T}\right\|_{2} \leqslant-\mu \lambda_{N} & \text { sticking: }\left\|\underline{\mathbf{u}}_{T}\right\|_{2}=0
\end{array}
$$

where $\mu \in[0,+\infty[$ is the friction coefficient. Assuming a radial evolution of the displacement field per time step, the velocity can be replaced with the increment of displacement in the previous law. 


\subsection{Weak formulation}

We define the following set of homogeneous kinematically admissible functions as:

$$
\underline{\mathbf{u}}(\underline{\mathbf{x}}, t) \in \mathcal{U}_{0}^{[0, T]}
$$

with $\mathcal{U}_{0}^{[0, T]}=\left\{\underline{\mathbf{u}}\right.$ has a finite energy and $\left.\forall(\underline{\mathbf{x}}, t) \in \partial_{1} \Omega \times[0, T], \underline{\mathbf{u}}(\underline{\mathbf{x}}, t)=\underline{\mathbf{0}}\right\}$

Equilibrium (3) is equivalent to the following integral formulation with compatibility of contact force field and displacement field to contact conditions (7) and (8):

$$
\forall \underline{\mathbf{u}}^{*} \in \mathcal{U}_{0}^{[0, T]}: \quad \int_{\Omega} \boldsymbol{\sigma}: \varepsilon\left(\underline{\mathbf{u}}^{*}\right) \mathrm{d} V-\int_{\partial_{2} \Omega} \underline{\mathbf{f}}_{\mathrm{ext}} \cdot \underline{\mathbf{u}}^{*} \mathrm{~d} S-\int_{\partial_{3} \Omega} \underline{\boldsymbol{\lambda}} \cdot \underline{\mathbf{u}}^{*} \mathrm{~d} S=0
$$

\subsection{Discretized weak formulation}

Using Hooke law for $\boldsymbol{\sigma}=\mathbb{K} \varepsilon$ and the finite element approximation for displacement field yield to the so-called equilibrium equation with respect to frictional contact conditions at each node:

$$
\forall \mathbf{u}^{*}: \int_{0}^{T} \mathbf{u}^{* T} \mathbf{K u} \mathrm{d} t=\int_{0}^{T} \mathbf{u}^{* T}\left(\mathbf{f}_{\mathrm{ext}}+\mathbf{f}_{\mathrm{ctc}}\right) \mathrm{d} t \quad \text { with } \quad\left\{\begin{array}{l}
\mathbf{v}=\mathbf{B u} \\
\mathbf{f}_{\mathrm{ctc}}=\mathbf{B}^{T} \boldsymbol{\lambda} \\
\mathcal{R}(\mathbf{v}, \boldsymbol{\lambda})=0
\end{array}\right.
$$

$\mathbf{K}$ is the stiffness matrix, $\mathbf{f}_{\text {ext }}$ and $\boldsymbol{\lambda}$ are generalized forces corresponding to $\underline{\mathbf{f}}_{\text {ext }}$ and $\underline{\boldsymbol{\lambda}}$, and $\mathbf{B}$ is a boolean matrix mapping the global vector of nodal values to the values on contact boundary nodes. All in all, we have to find displacement field $\mathbf{u}$ and contact force field $\mathbf{f}_{\text {ctc }}$ verifying (11) for all weight functions $\mathbf{u}^{*}$.

Time interval is discretized into a subsequent of time steps $t_{0 \leqslant k \leqslant m}$ describing a regular time stepping, i.e. $t_{k+1}=t_{k}+\Delta t$.

\subsection{Reduced weak formulation and a posteriori analysis}

Assuming $\mathbf{u}(\mathbf{x}, t)$ being the solution of the discretized reference problem depicted in section 2 defined over the whole space (including boundaries) and time domains, the corresponding stress field is denoted by $\boldsymbol{\sigma}(\mathbf{x}, t)$ and the contact force field by $\boldsymbol{\lambda}(\mathbf{x}, t)$.

Discretized nodal displacements (with $n$ degrees of freedom) are known for all time steps $t_{0 \leqslant k \leqslant m}$ and are cast into a snapshot matrix defined as follows:

$$
\mathbf{U}=\left[\begin{array}{cccc}
\mathbf{u}\left(x_{1}, t_{0}\right) & \mathbf{u}\left(x_{1}, t_{1}\right) & \cdots & \mathbf{u}\left(x_{1}, t_{m}\right) \\
\mathbf{u}\left(x_{2}, t_{0}\right) & \mathbf{u}\left(x_{2}, t_{1}\right) & \cdots & \mathbf{u}\left(x_{2}, t_{m}\right) \\
\vdots & \vdots & \ddots & \vdots \\
\mathbf{u}\left(x_{n}, t_{0}\right) & \mathbf{u}\left(x_{n}, t_{1}\right) & \cdots & \mathbf{u}\left(x_{n}, t_{m}\right)
\end{array}\right]=\left[\begin{array}{llll}
\mathbf{w}\left(t_{0}\right) & \mathbf{w}\left(t_{1}\right) & \cdots & \mathbf{w}\left(t_{m}\right)
\end{array}\right]
$$


$\mathbf{U} \in \mathbb{R}^{n \times m}$ is a real rectangular matrix. According to the singular value theorem [33], U can be factorized using singular value decomposition (SVD):

$$
\mathbf{U}=\boldsymbol{\Upsilon} \boldsymbol{\Sigma} \boldsymbol{\Phi}^{T}=\left[\begin{array}{lll}
\boldsymbol{\Upsilon}_{1} & \cdots & \boldsymbol{\Upsilon}_{n}
\end{array}\right]\left[\begin{array}{cccc}
\sigma_{1} & 0 & \cdots & 0 \\
0 & \sigma_{2} & & \vdots \\
\vdots & & \ddots & 0 \\
0 & \cdots & 0 & \sigma_{r} \\
\mathbf{0} & \cdots & \mathbf{0} & \mathbf{0}
\end{array}\right]\left[\begin{array}{c}
\boldsymbol{\Phi}_{1}^{T} \\
\vdots \\
\boldsymbol{\Phi}_{m}^{T}
\end{array}\right]
$$

with $r=\min (n, m), \Upsilon \boldsymbol{\Upsilon} \in \mathbb{R}^{n \times n}$ is a unitary matrix containing left-singular vectors, $\boldsymbol{\Phi} \in \mathbb{R}^{m \times m}$ a unitary matrix containing right-singular vectors and $\boldsymbol{\Sigma} \in \mathbb{R}^{n \times m}$ contains positive singular values $\sigma_{i}$ in increasing order.

Moreover, left-singular vectors $\boldsymbol{\Upsilon}_{i}$ are space vectors which are combined with time functions $\boldsymbol{\Phi}_{i}$ scaled with the singular value $\sigma_{i}$. Consequently, displacement field can be written as a sum of radial solutions:

$$
\mathbf{U}=\boldsymbol{\Upsilon}_{1} \sigma_{1} \boldsymbol{\Phi}_{1}^{T}+\boldsymbol{\Upsilon}_{2} \sigma_{2} \boldsymbol{\Phi}_{2}^{T}+\cdots+\underbrace{\Upsilon_{\text {radial solution }} \sigma_{i} \boldsymbol{\Phi}_{i}^{T}}_{i^{\text {th }}}+\cdots+\boldsymbol{\Upsilon}_{r} \sigma_{r} \boldsymbol{\Phi}_{r}^{T}
$$

This decomposition is unique up to an arbitrary sign for the couples of vectors $\left(\mathbf{\Upsilon}_{i}, \boldsymbol{\Phi}_{i}\right)$.

Given $\mathbf{U}$ and its SVD, and taking into account only $K \leq r$ first singular values, allows to define an approximation $\check{\mathbf{U}}$ such that:

$$
\check{\mathbf{U}}=\mathbf{\Upsilon}_{1} \sigma_{1} \boldsymbol{\Phi}_{1}^{T}+\mathbf{\Upsilon}_{2} \sigma_{2} \boldsymbol{\Phi}_{2}^{T}+\cdots+\Upsilon_{K} \sigma_{K} \boldsymbol{\Phi}_{K}^{T}
$$

According to the Eckart-Young's low rank approximation theorem [33], Uั is the best approximation of rank $K$ of $\mathbf{U}$ according to the Frobenius' norm. It corresponds to the discrete POD of $\mathbf{U}$. Moreover, if $K=r$ then $\check{\mathbf{U}}=\mathbf{U}$.

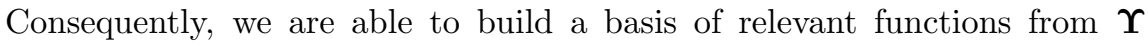
containing the $K$ first left-singular space vectors. Moreover, the first vectors are the most energetic ones (i.e. they describe globally the solution and contain a great fraction of the elastic energy), as it will be exemplified in the following.

The elastic energy of the displacement field solution integrated over the studied time interval is given by

$$
E=\frac{1}{2} \int_{0}^{T} \mathbf{w}(t)^{T} \mathbf{K} \mathbf{w}(t) \mathrm{d} t
$$

It can be distributed among radial solutions as $E=\sum_{i=1}^{r} E_{i}$ where

$$
E_{i}=\frac{\sigma_{i}^{2}}{2} \mathbf{\Upsilon}_{i}^{T} \mathbf{K} \Upsilon_{i} \int_{0}^{T} \boldsymbol{\Phi}_{i}^{T} \boldsymbol{\Phi}_{i} \mathrm{~d} t
$$

corresponds to the elastic energy contained in the $i^{\text {th }}$ radial solution.

\section{Application to 2D frictional contact problems}

Though frictional contact problems lead to non-smooth solutions, the representation of a solution in the space-time domain allows to define a reduced basis 


\begin{tabular}{|c|c|c|c|c|}
\cline { 3 - 5 } \multicolumn{2}{c|}{} & Case A & Case B & Case C \\
\hline Number of time steps & $m$ & 201 & 201 & 185 \\
\hline Number of dofs & $n$ & 1144 & 2552 & 2552 \\
\hline Number of contacting nodes & $n_{C}$ & 101 & 101 & 101 \\
\hline Young modulus & $E$ & \multicolumn{3}{|c|}{$3000 \mathrm{~Pa}$} \\
\hline Poisson ratio & $\nu$ & \multicolumn{3}{|c|}{0.3} \\
\hline Friction coefficient & $\mu$ & \multicolumn{3}{|c}{0.15} \\
\hline Final time & $T$ & \multicolumn{3}{c}{$1 \mathrm{~s}$} \\
\hline
\end{tabular}

Table 1: Simulation parameters.

suited to approximate it. The following examples are aimed to exemplify this on $2 \mathrm{D}$ frictional contact problems once their space-time solutions have been obtained. The first test model is a single contact case, the second one represents a larger structure when a part of it encounters contact conditions while the last one uses a more complex loading which is known to be less easily represented on a reduced radial base. Each time, we discuss the contribution of the different SVD modes and of their scale lengths.

Linear 3-node triangular finite elements are used for the spatial discretization.

Material properties, simulation characteristics and parameters are given in table 1 , for the upcoming three cases.

\subsection{Hemicylindrical body contacting a rigid basement (case}

A)

The first model (figure 2) consists in an elastic hemicylindrical body contacting a frictional rigid basement. We prescribe displacement on the upper boundary such that a large contact zone occurs. The scale of the structure and the scale of the contact zone are similar. Initially, the solid is not contacting. From time-step $t_{1}$ to $t_{40}$, it approaches and becomes pressed on the rigid basement. Meanwhile, a tangential displacement is prescribed to activate a global frictional reaction at the contacting zone.

We apply SVD to displacement field in order to generate singular vectors in space (figure 4) and in time (figure 3). We notice that for this case a few couple of modes ( 4 or 5 ) are needed to render $99.9 \%$ of the elastic energy of the highly reducible solution, which is therefore provided that we consider the space-time evolution in a while.

On figure 4, we notice that the three first modes refer to a large scale contribution acting on the whole body. Whereas after the fourth one, space modes are remarkably localized in the vicinity of contact area. After the $6^{\text {th }}$ mode, the characteristic length scale of the mode get closer and closer to the discretization length scale (i.e. the mesh size). Similarly, respective time functions have a global / local behavior on the time interval. 


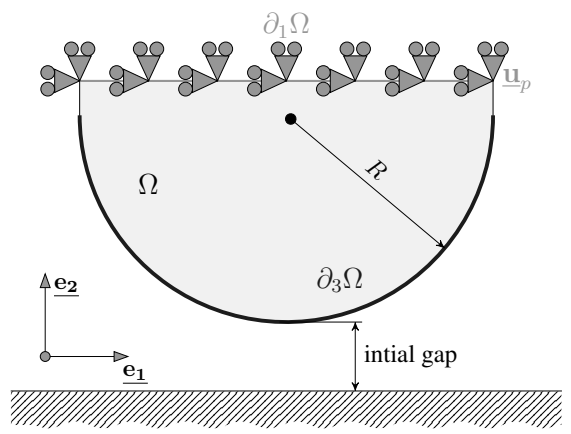

(a)

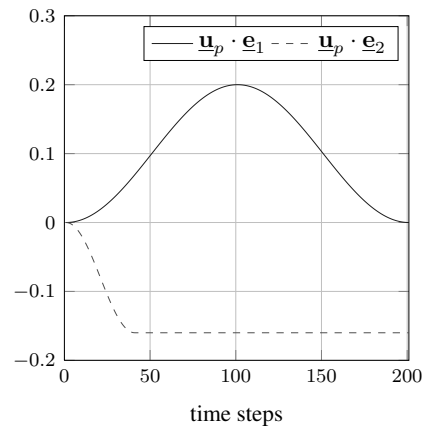

(b)

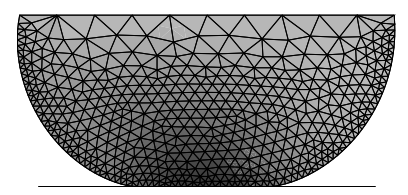

(c)

Figure 2: (a) Model - (b) Prescribed displacement evolution - (c) Shape of the deformed body at $t_{50}$.

\subsection{Cantilevered indenter contacting a rigid basement (case B)}

The second problem (figure 5) consists in an elastic clamped cantilevered hemicylindrical indenter contacting a frictional rigid basement. External loads are designed in order to get different status at the contacting nodes during the studied time interval (no contact, sliding, sticking). The size of the effective contact area is smaller than the scale of the structure.

In the same way, by applying the SVD to the displacement field, we get time and space functions. A few couple of modes (4 or 5 ) are also needed to render $99.9 \%$ of the elastic energy of the simulation (figure 6). The solution of this second problem is also highly reducible.

From figures 6 and 7, same conclusions as for the first simulation case can be drawn. Status switching seems to not affect reducibility of the solution. For this problem, the length scale of the global structure is more separated from the one of the contact area, and this is also depicted in the succession of spatial modes.

\subsection{Cantilevered indenter contacting a rigid basement with a non radial external load (case $\mathrm{C}$ )}

The last problem (figure 8) is the same as the second excepting for the external loads. We design a mobile half-sine lobe load moving along the upper boundary of the body with an increasing amplitude. It is well-known that this kind of solicitation is not efficiently described by a finite set of radial functions (15). A soft contact occur at the bottom of the hemicylindrical part.

In figure 8 , we introduce the space variable $\ell$ localizing he position of the 


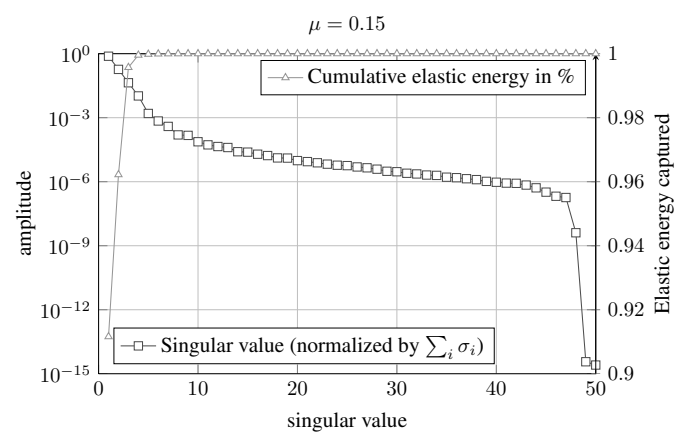

(a)

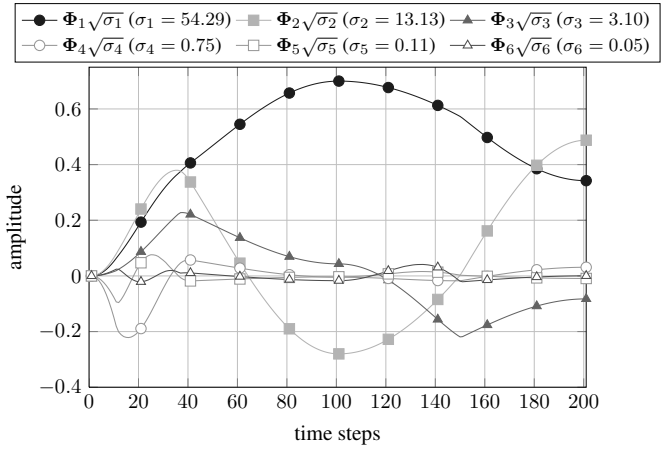

(b)

Figure 3: (a) The a posteriori analysis of the first 50 singular values and the cumulative elastic energy of the modes - (b) The a posteriori analysis of the first 6 right-singular time modes $\boldsymbol{\Phi}_{i}$ scaled by $\sqrt{\sigma_{i}}$. 

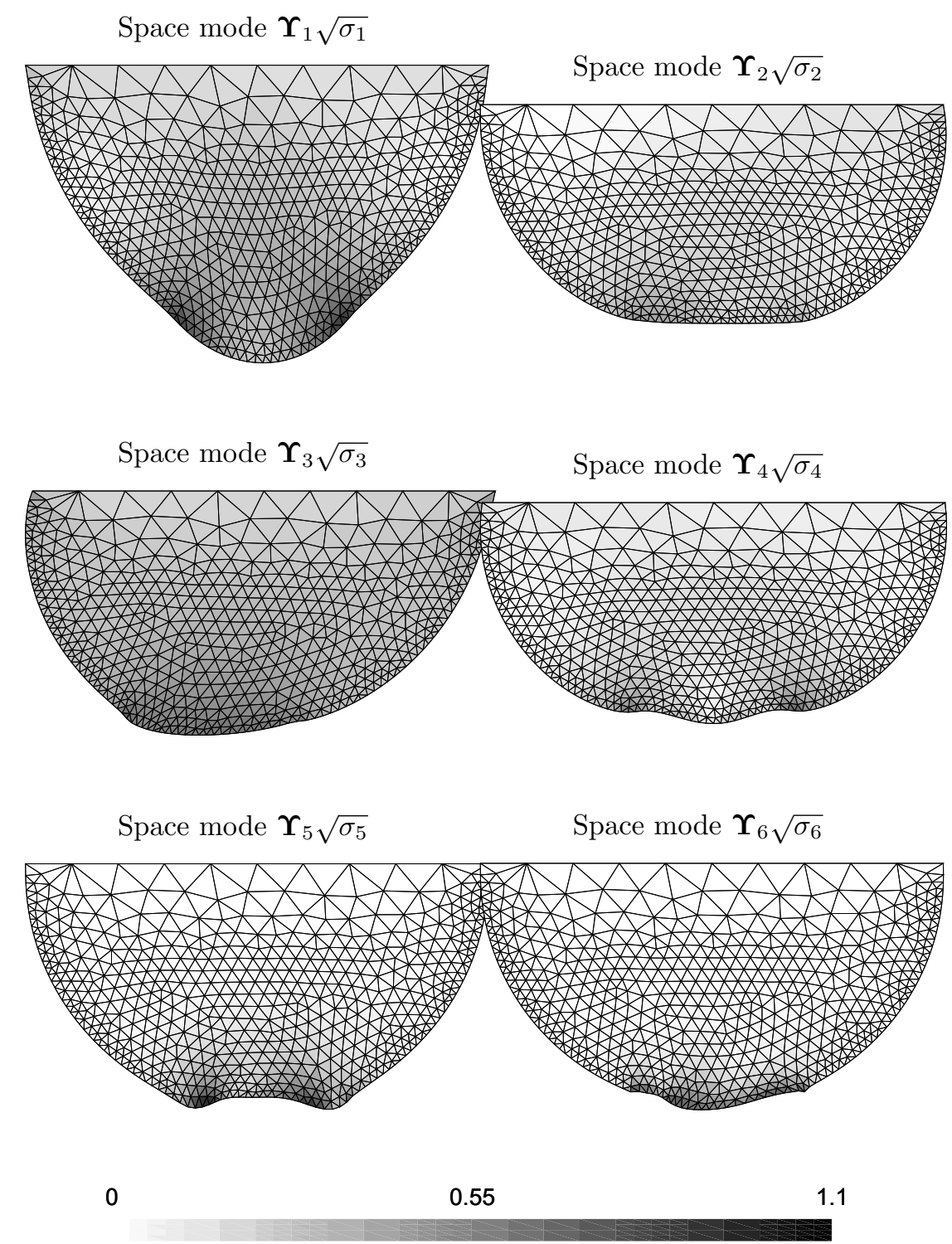

Figure 4: Shapes of deformed body for the left-singular space modes scaled by respective $\sqrt{\sigma}$ and the grayscale color map depicts the gradient of the amplitude of the displacement field. 


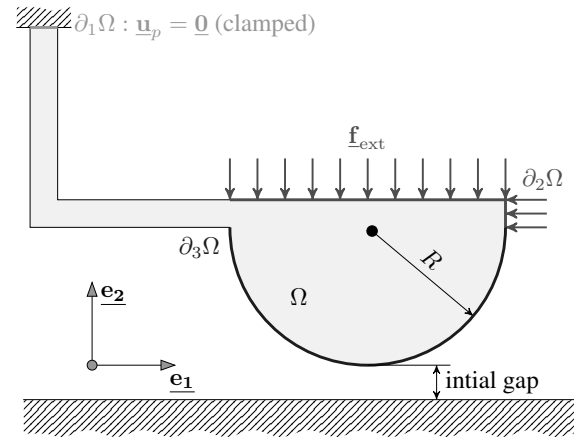

(a)

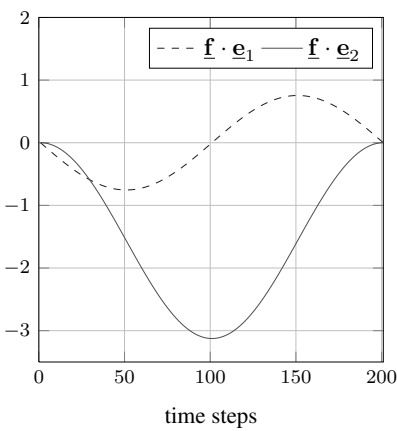

(b)

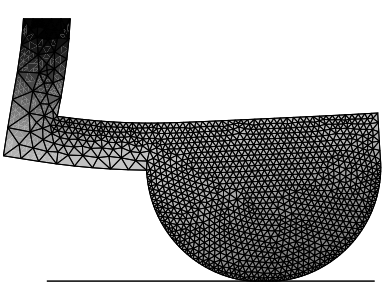

(c)

Figure 5: (a) Model - (b) External loads evolutions. Initially, the solid is not contacting. - (c) Shape of the deformed body at $t_{50}$.

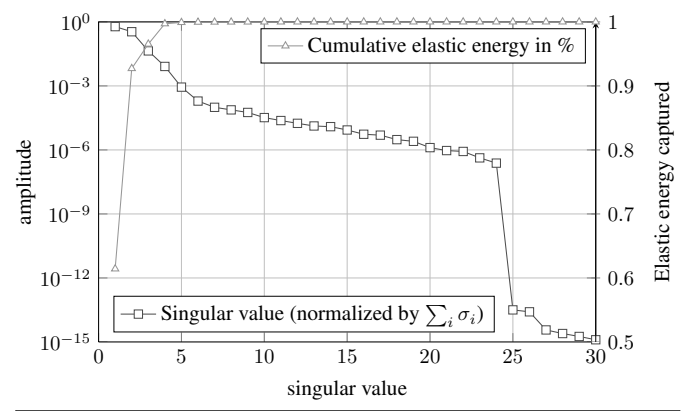

$\longrightarrow \boldsymbol{\Phi}_{1} \sqrt{\sigma_{1}}\left(\sigma_{1}=23.65\right)-\boldsymbol{\Phi}_{2} \sqrt{\sigma_{2}}\left(\sigma_{2}=13.63\right) \longrightarrow \boldsymbol{\Phi}_{3} \sqrt{\sigma_{3}}\left(\sigma_{3}=1.72\right)$

$\boldsymbol{\Phi}_{4} \sqrt{\sigma_{4}}\left(\sigma_{4}=0.32\right)-\square-\boldsymbol{\Phi}_{5} \sqrt{\sigma_{5}}\left(\sigma_{5}=0.034\right)-\triangle-\boldsymbol{\Phi}_{6} \sqrt{\sigma_{6}}\left(\sigma_{6}=0.0077\right)$

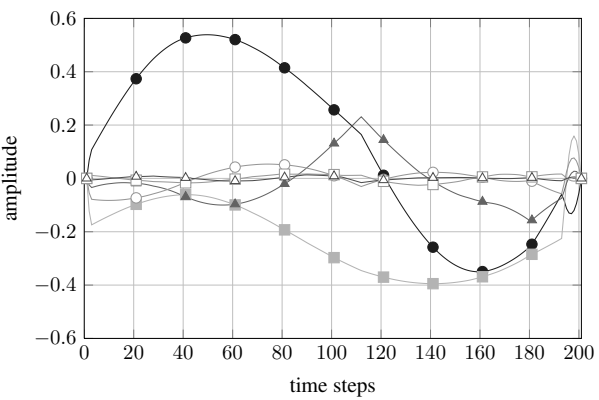

Figure 6: (a) The a posteriori analysis of the first 50 singular values and the cumulative elastic energy of the modes - (b) The a posteriori analysis of the first 6 right-singular time modes $\boldsymbol{\Phi}_{i}$ scaled by $\sqrt{\sigma_{i}}$. 


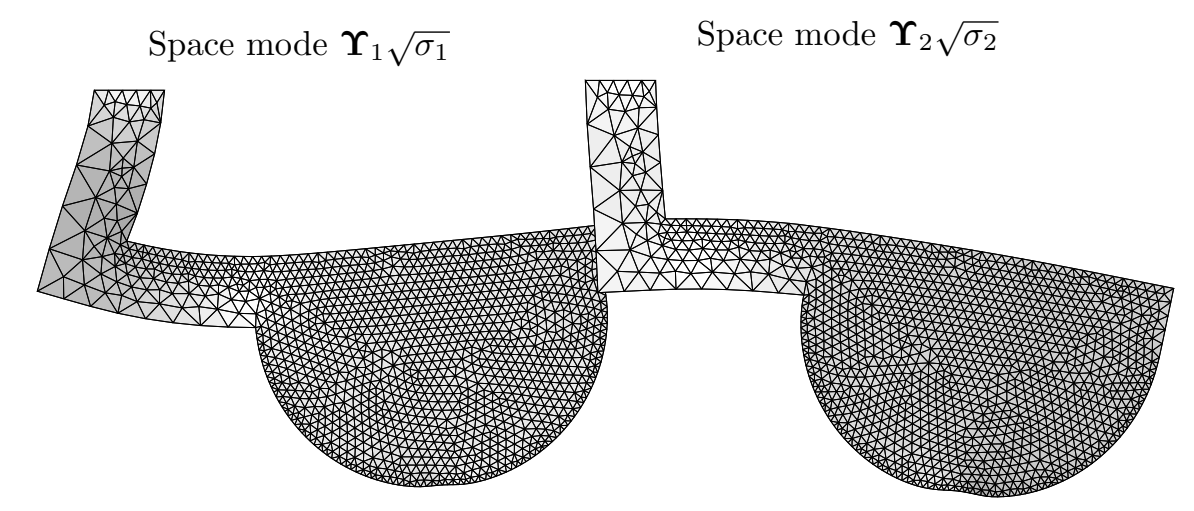

Space mode $\boldsymbol{\Upsilon}_{3} \sqrt{\sigma_{3}} \quad$ Space mode $\boldsymbol{\Upsilon}_{4} \sqrt{\sigma_{4}}$

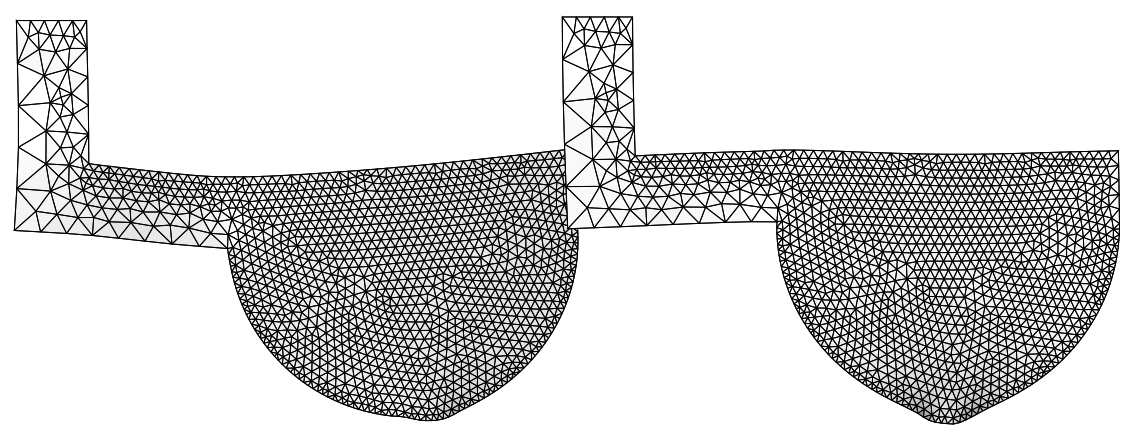

Space mode $\boldsymbol{\Upsilon}_{5} \sqrt{\sigma_{5}} \quad$ Space mode $\boldsymbol{\Upsilon}_{6} \sqrt{\sigma_{6}}$

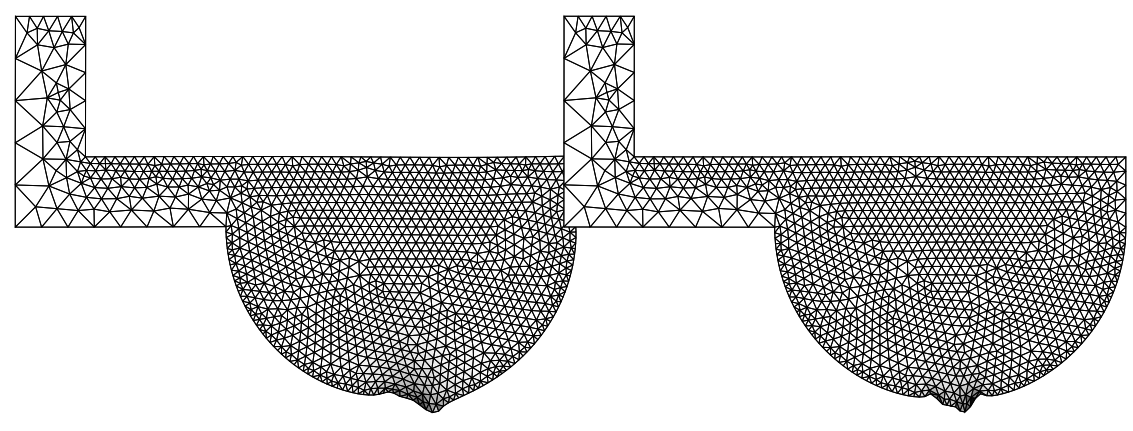

0

0.9

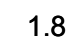

Figure 7: Shapes of deformed body for the left-singular space modes scaled by respective $\sqrt{\sigma}$ and the grayscale color map depicts the gradient of the amplitude of the displacement field. 


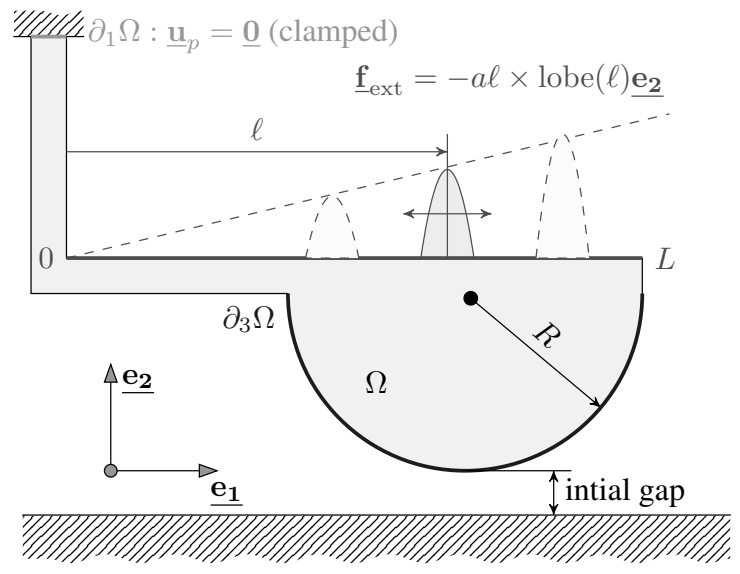

(a)

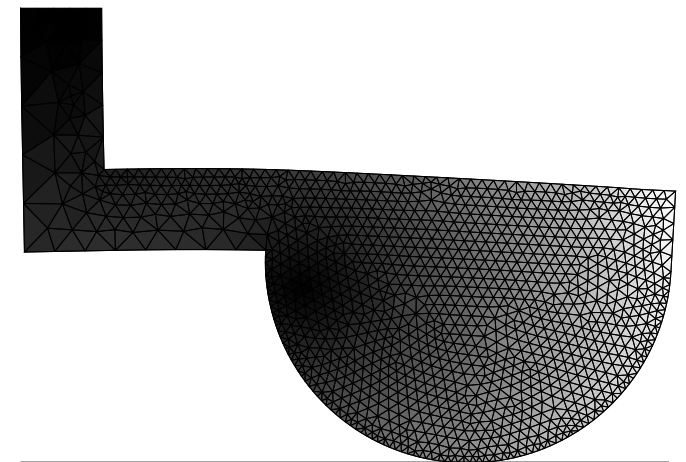

(b)

Figure 8: Model C - (a) Model with a mobile external load. The load is a half-sine moving twice from $\ell=0$ to $\ell=L$ and back to $\ell=0$. Its amplitude varies linearly according to $\ell$. At $\ell=0$ external load are null - (b) Shape of the deformed body when $\ell=L$.

lobe on the upper boundary. The evolution of $\ell$ according the time variable is prescribed as follows:

$$
\left\{\begin{array}{lll}
t \in\left[0, \frac{T}{4}\right]: & & \ell=4 L \frac{t}{T} \\
t \in\left[\frac{T}{4}, \frac{T}{2}\right]: & & \ell=4 L\left(1-\frac{t}{T}\right) \\
t \in\left[\frac{T}{2}, \frac{3 T}{4}\right]: & \ell=4 L \frac{t}{T} \\
t \in\left[\frac{3 T}{4}, T\right]: & \ell=4 L\left(1-\frac{t}{T}\right)
\end{array}\right.
$$

According to figure 9, the reduction of the solution is clearly less efficient than other problems. Twenty modes are needed (instead of five) to contain $99.9 \%$ of the elastic energy. Space modes on figure 10 are concerned with three scales of the problem: the body length scale, the contact zone length and the load length scale (the wavelength of the lobe) on the upper boundary where it is applied. 


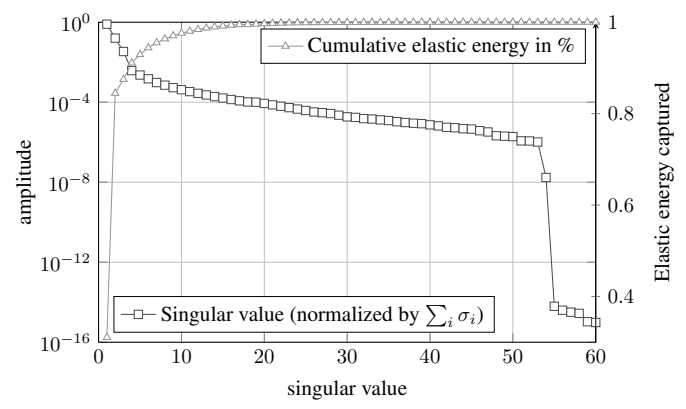

(a)

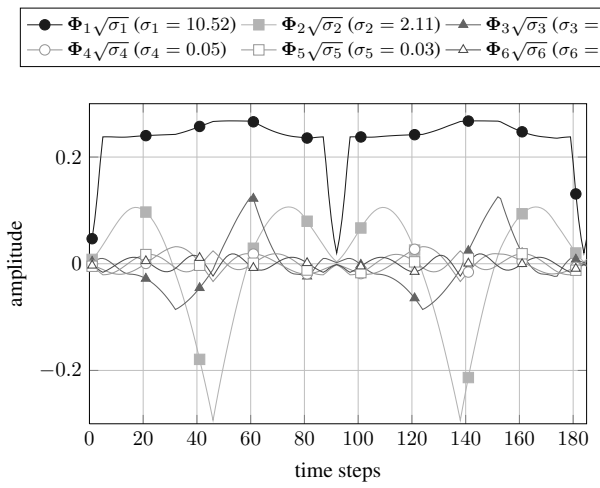

(b)

Figure 9: (a) The a posteriori analysis of the first 50 singular values and the cumulative elastic energy through modes - (b) The a posteriori analysis of the first 6 right-singular time modes $\boldsymbol{\Phi}_{i}$ scaled by $\sqrt{\sigma_{i}}$. 
Space mode $\boldsymbol{\Upsilon}_{1} \sqrt{\sigma_{1}}$

Space mode $\boldsymbol{\Upsilon}_{2} \sqrt{\sigma_{2}}$

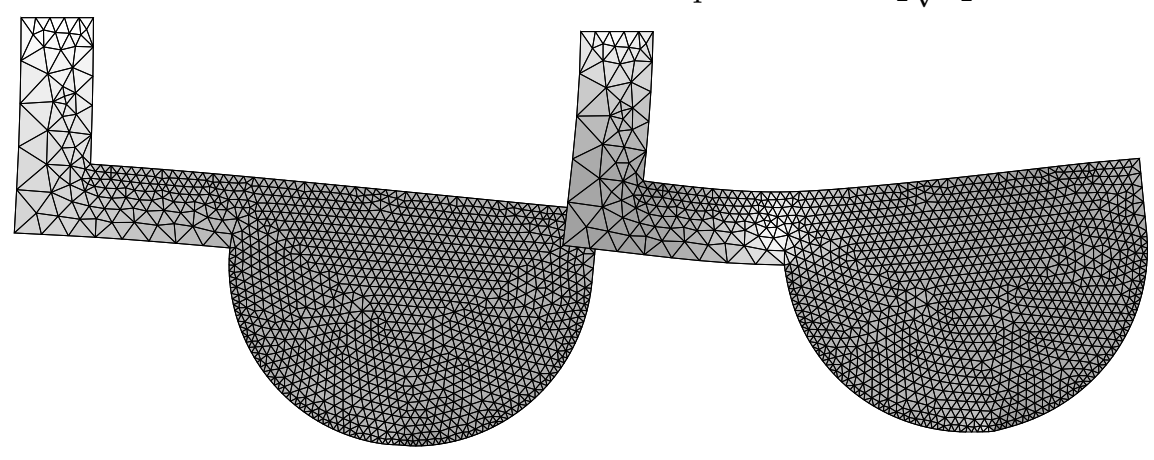

Space mode $\boldsymbol{\Upsilon}_{3} \sqrt{\sigma_{3}}$

Space mode $\boldsymbol{\Upsilon}_{4} \sqrt{\sigma_{4}}$

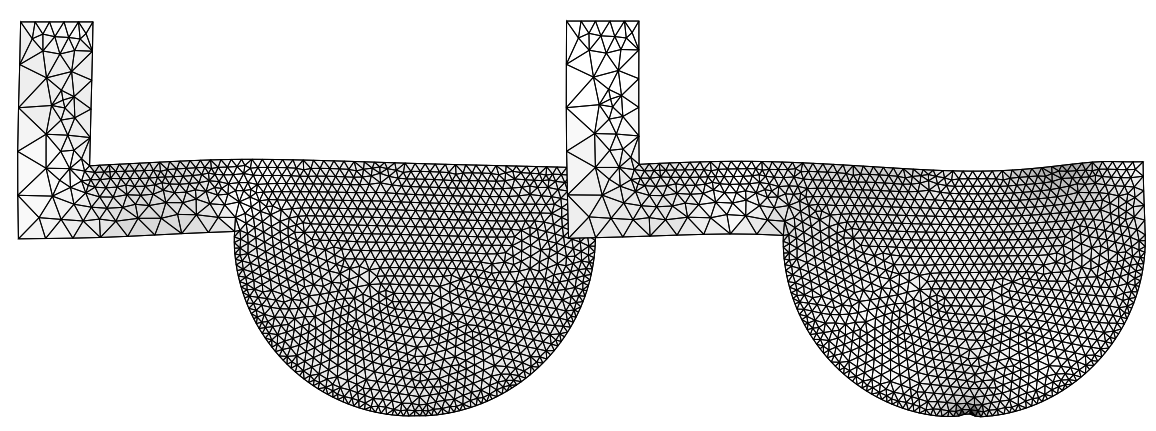

Space mode $\boldsymbol{\Upsilon}_{5} \sqrt{\sigma_{5}}$

Space mode $\Upsilon_{6} \sqrt{\sigma_{6}}$

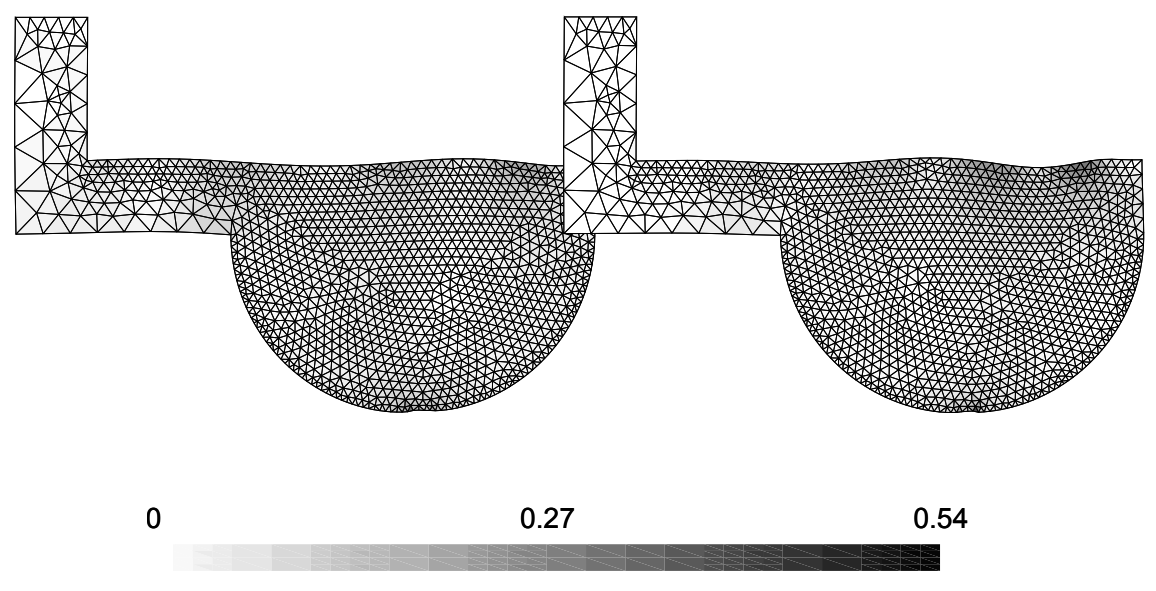

Figure 10: Shapes of deformed body depicts the left-singular space modes scaled by respective $\sqrt{\sigma}$ and the grayscale color map depicts the gradient of the amplitude of the displacement field. 


\subsection{SVD functions and multiscale contents}

Time-space model reduction emphasizes scale separability phenomenon. At least, two scales of the problem can be distinguished: the global scale of the structure and a local scale where contact occurs. First radial solutions obtained by SVD describe globally the displacement field (both in space but also in time) whereas higher order modes bring corrections enhancing precision at contact interface.

We can notice that time-space modes can be dominated by specific solicitations occurring during the deformation process. It is clear in the model A : the first and the second modes are linked to the compression on the rigid basement whereas the third one is linked to shearing effects involved by friction: the appropriate linear combination of these three modes for each time step covers roughly $95 \%$ of the elastic energy involved. Nevertheless and due to orthogonality properties and energetic considerations, SVD modes are not prescribed to be characterized by load components. Consequently, we have to distinguish SVD decomposition and linear superposition principle even though both span the exact solution.

\section{Efficient resolution using reduced order model}

Scale separability observation encourages the deployment of multiscale strategies [34]. Generally speaking, they consist in distinguishing a global behavior occurring on the whole structure and a behavior localized on specific area. For instance, in [35], a cracked body is studied taking into account friction contacts between crack faces. Behavior at interfaces (crack faces) is treated as an autonomous problem (own discretization, constitutive law, internal variables) and then linked in a weak sense to the global problem. In [36], a local/global strategy is used by solving the global problem in a reduced space (obtained by POD) while the local problem (where localized damage phenomenon occurs) is fully resolved. Then, both of the global and local problems are coupled. Other efficient approaches are multigrid solvers suitable for various non-linear problems [37, 38, 39, 40]. For frictional contact problems, multigrid methods were proposed in [22]. Various iterative solvers (called "smoothers") have a remarkable smoothing effect on high-frequency component of the error (relatively to the discretization). In contrast, low-frequency of the error is slowly damped by these solvers. Consequently, coarser discretization are introduced (coarse grids) to bring corrections to fine grids to increase convergence rate.

We can draw an analogy between the previous observations and multigrid solvers. We propose to consider global scale modes as coarse grid components. In the same way as multigrid methods, we try to bring time-space corrections from a subspace spanned with these first space modes obtained by an a posteriori analysis. Corrections are quickly computed on a reduced order model (defined by a reduced basis built on left-singular $\Upsilon$ vectors of SVD). Then, these corrections are transferred on the full order model (full dimensional model) to increase convergence rate of the iterative LATIN solver [27] (considered as the smoother). In this study we rely on the SVD modes that are precomputed to exemplify these effects on the solvers. Of course, such modes, taking into account their scale properties could have been computed by other means (for instance, coarse grid 
computations, analytical local solutions ...) but this is out of the scope of this article.

\subsection{LATIN method}

One of the major points of our strategy is the non-incremental LATIN method introduced by Ladevèze and designed for time-dependent problems. Champaney et al. in $[41,28,42]$ used this method to solve problems involving contact between substructures. In [43, 44], LATIN method is used to solve 3D impacts with frictional contacts with plasticity interfaces. Herein, we give a short recall of the LATIN method directly formulated for frictional contact problems with a two-field weak formulation. Stabilized formulations (three-field weak formulation with stabilization operator) can be used as in [45].

LATIN method is based on three principles illustrated on figure 11:

(P1) Separation of the linear and non-linear behavior. $\mathcal{A}$ denotes the set of solutions $\mathbf{s}=(\mathbf{v}, \boldsymbol{\lambda})$ satisfying linear constitutive law, kinematic admissibility and static admissibility. These are defined on the whole time-space domains $\Omega \times[0, T] . \quad \Gamma$ denotes the set of solutions $\hat{\mathbf{s}}=(\hat{\mathbf{v}}, \hat{\boldsymbol{\lambda}})$ verifying frictional contact conditions and are defined locally at the contacting interface and on the whole time interval $\partial_{3} \Omega \times[0, T]$. The solution of the problem is $\mathbf{s} \in \mathcal{A} \cap \boldsymbol{\Gamma}$.

(P2) A two-staged iterative algorithm. The solution of the problem is searched with the construction of two sequences of approximations belonging alternatively to $\mathcal{A}$ and then $\boldsymbol{\Gamma}$. At the $i^{\text {th }}$ iteration, global stage consists in finding $\mathbf{s}_{i+1}=\left(\mathbf{v}_{i+1}, \boldsymbol{\lambda}_{i+1}\right) \in \mathcal{A}$ with a search direction $\left(\mathbf{s}_{i+1}-\hat{\mathbf{s}}_{i}\right)=$ $\left(\mathbf{v}_{i+1}-\hat{\mathbf{v}}_{i}, \boldsymbol{\lambda}_{i+1}-\hat{\boldsymbol{\lambda}}_{i}\right) \in \mathbf{E}^{-}$. Note that $\hat{\mathbf{s}}_{i}=\left(\hat{\mathbf{v}}_{i}, \hat{\boldsymbol{\lambda}}_{i}\right)$ is known at this stage. Then, local stage consists in finding $\hat{\mathbf{s}}_{i+1}=\left(\hat{\mathbf{v}}_{i+1}, \hat{\boldsymbol{\lambda}}_{i+1}\right) \in \boldsymbol{\Gamma}$ with another search direction $\left(\hat{\mathbf{s}}_{i+1}-\mathbf{s}_{i+1}\right)=\left(\hat{\mathbf{v}}_{i+1}-\mathbf{v}_{i+1}, \hat{\boldsymbol{\lambda}}_{i+1}-\boldsymbol{\lambda}_{i+1}\right) \in \mathbf{E}^{+}$. And $\mathbf{s}_{i+1}=\left(\hat{\mathbf{v}}_{i+1}, \hat{\boldsymbol{\lambda}}_{i+1}\right)$ is known from the previous stage.

(P3) Radial approximation. The unknowns are represented as a sum of products between a time function and a space functions. This principle is not use herein.

To specify the equations to solve at global and local stage, we use a displacement formulation. Velocity formulation well-suited for dynamical framework could also be used [43].

Global stage. This stage consists in finding a displacement field $\mathbf{v}(\mathbf{x}, t)$ and a contact force field $\boldsymbol{\lambda}(\mathbf{x}, t)$ defined over $\partial_{3} \Omega \times[0, T]$ verifying the balance of the solid with the search direction (19).

$$
\begin{aligned}
\left(\mathbf{E}^{-}\right): \boldsymbol{\lambda} & -\hat{\boldsymbol{\lambda}}=-\mathbf{k}(\mathbf{v}-\hat{\mathbf{v}}) \\
& \Leftrightarrow\left\{\begin{array}{c}
\boldsymbol{\lambda}_{T} \\
\boldsymbol{\lambda}_{N}
\end{array}\right\}-\left\{\begin{array}{c}
\hat{\boldsymbol{\lambda}}_{T} \\
\hat{\boldsymbol{\lambda}}_{N}
\end{array}\right\}=-\left[\begin{array}{cc}
k_{T} \mathbf{I}_{\mathbf{d}} & \mathbf{0} \\
\mathbf{0} & k_{N} \mathbf{I}_{\mathbf{d}}
\end{array}\right]\left(\left\{\begin{array}{c}
\mathbf{v}_{T} \\
\mathbf{v}_{N}
\end{array}\right\}-\left\{\begin{array}{l}
\hat{\mathbf{v}}_{T} \\
\hat{\mathbf{v}}_{N}
\end{array}\right\}\right)
\end{aligned}
$$

$\mathbf{I}_{\mathbf{d}}$ is the identity matrix of size equal to the number of contacting nodes. $k_{N}$ and $k_{T}$ are parameters of the method defined at each node. It is similar to the 


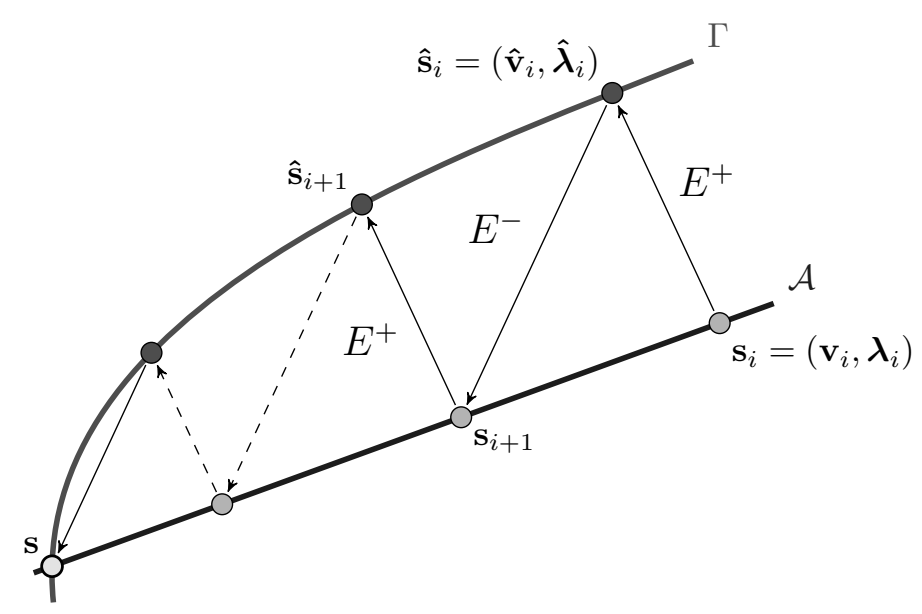

Figure 11: LATIN non-linear iterative solver.

penalty parameter in an augmented Lagrangian formulation. Different values for the normal and tangential problem can be chosen. These values influence only on the convergence rate and optimal values are related to mechanical properties of the studied body [27, 42, 46, 45]. Generally speaking and according to [46], optimum values are searched as follows:

$$
k=E L_{c}
$$

with $E$ the Young's modulus and $L_{c}$ a contact characteristic dimension. $k$ has the dimension of a stiffness. A parametric study was carried out for case A (figure 12) to identify the optimal value of $k$ for both LATIN and FAS/LATIN algorithm (algorithm proposed). (21).

Displacement field and contact force field verifying equilibrium are given by

$$
\left\{\begin{array}{l}
\mathbf{K u}=\mathbf{f}_{\text {ext }}+\mathbf{B}^{T} \boldsymbol{\lambda} \\
\mathbf{v}=\mathbf{B u} \\
\boldsymbol{\lambda}=\hat{\boldsymbol{\lambda}}-\mathbf{k}(\mathbf{v}-\hat{\mathbf{v}})
\end{array}\right.
$$

Local stage. The local stage is an updating stage for the contacting interface. Its solution is explicit and is deduced from search direction (22) and non-linear contact conditions. 

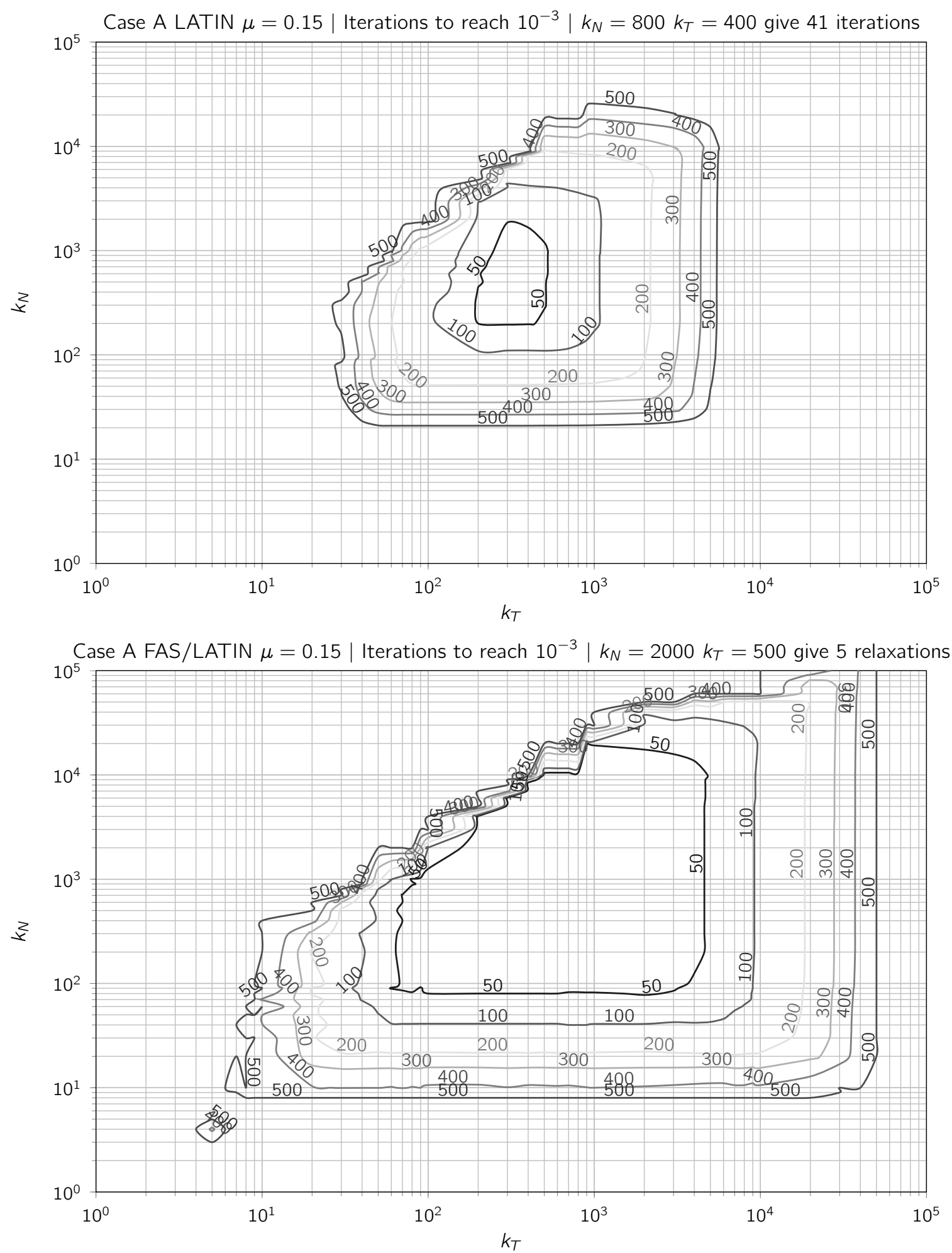

Figure 12: Number of LATIN iterations to reach a precision of $10^{-3}$ according different value for search directions (normal $k_{N}$ and tangential $k_{T}$ problem). We can remark that FAS/LATIN doesn't mgdify the optimal value and spreads the "optimal zone" 


\begin{tabular}{|c|c|}
\hline \multicolumn{2}{|c|}{ Normal components } \\
\hline \multicolumn{2}{|c|}{$\hat{\lambda}_{N}=\left\langle c_{N}\right\rangle_{-} \quad \hat{v}_{N}=j-\frac{1}{k_{N}}\left\langle c_{N}\right\rangle_{+}$} \\
\hline \multicolumn{2}{|c|}{ Tangential components } \\
\hline Sliding: $\left\|\mathbf{c}_{T}\right\|_{2}>\mu\left|\hat{\lambda}_{N}\right|$ & Sticking: $\left\|\mathbf{c}_{T}\right\|_{2} \leqslant \mu\left|\hat{\lambda}_{N}\right|$ \\
\hline $\mathbf{t}=\mathbf{c}_{T} /\left\|\mathbf{c}_{T}\right\|_{2}$ & $\hat{\boldsymbol{\lambda}}_{T}=\mathbf{c}_{T}$ \\
$\hat{\boldsymbol{\lambda}}_{T}=\mu\left|\hat{\lambda}_{N}\right| \mathbf{t}$ & $\hat{\mathbf{v}}_{T}^{k}=\hat{\mathbf{v}}_{T}^{k-1}$ \\
$\hat{\mathbf{v}}_{T}^{k}=\hat{\mathbf{v}}_{T}^{k-1}-\frac{1}{k_{T}}\left(\left\|\mathbf{c}_{T}\right\|_{2}-\mu\left|\hat{\lambda}_{N}\right|\right) \mathbf{t}$ & \\
\hline
\end{tabular}

Table 2: Solutions of the local stage.

$$
\begin{aligned}
\left(\mathbf{E}^{+}\right): \hat{\boldsymbol{\lambda}}-\boldsymbol{\lambda} & =\mathbf{k}(\hat{\mathbf{v}}-\mathbf{v}) \\
& \Leftrightarrow\left\{\begin{array}{c}
\hat{\boldsymbol{\lambda}}_{T} \\
\hat{\boldsymbol{\lambda}}_{N}
\end{array}\right\}-\left\{\begin{array}{c}
\boldsymbol{\lambda}_{T} \\
\boldsymbol{\lambda}_{N}
\end{array}\right\}=\left[\begin{array}{cc}
k_{T} \mathbf{I}_{\mathbf{d}} & \mathbf{0} \\
\mathbf{0} & k_{N} \mathbf{I}_{\mathbf{d}}
\end{array}\right]\left(\left\{\begin{array}{c}
\hat{\mathbf{v}}_{T} \\
\hat{\mathbf{v}}_{N}
\end{array}\right\}-\left\{\begin{array}{c}
\mathbf{v}_{T} \\
\mathbf{v}_{N}
\end{array}\right\}\right)
\end{aligned}
$$

Equivalently, search directions can be rewritten for both tangential and normal contact. For each contacting node, the search direction yields to equations (23) and (24). $\mathbf{v}_{T}^{k}$ refers to the nodal displacement on the contacting interface in the tangential plane at time step $t_{k}$.

$$
\begin{gathered}
c_{N}=\lambda_{N}-k_{N}\left(v_{N}-j\right)=\hat{\lambda}_{N}-k_{N}\left(\hat{v}_{N}-j\right) \\
\mathbf{c}_{T}=\boldsymbol{\lambda}_{T}-k_{T}\left(\mathbf{v}_{T}^{k}-\hat{\mathbf{v}}_{T}^{k-1}\right)=\hat{\boldsymbol{\lambda}}_{T}-k_{T}\left(\hat{\mathbf{v}}_{T}^{k}-\hat{\mathbf{v}}_{T}^{k-1}\right)
\end{gathered}
$$

Solutions of the local stage are given in table 2 and have to be computed for each node belonging to the contacting interface and for each time step.

Initialization and stopping criterion. To initiate the iterative process, a linear contactless prediction is computed for $\mathbf{s}_{0}$. We use stopping criterion (25) introduced in [47]. Iterative process is stopped once $\mathcal{I}<\varepsilon$.

$$
\begin{aligned}
\mathcal{I}=\max \left(\sqrt{\frac{\left\|\mathbf{s}_{N}-\hat{\mathbf{s}}_{N}\right\|_{\infty}^{2}}{\left\|\mathbf{s}_{N}\right\|_{\infty}^{2}+\left\|\hat{\mathbf{s}}_{N}\right\|_{\infty}^{2}}}, \sqrt{\frac{\left\|\mathbf{s}_{T}-\hat{\mathbf{s}}_{T}\right\|_{\infty}^{2}}{\left\|\mathbf{s}_{T}\right\|_{\infty}^{2}+\left\|\hat{\mathbf{s}}_{T}\right\|_{\infty}^{2}}}\right) \\
\text { with }\left\|\mathbf{s}_{N, T}\right\|_{\infty}^{2}=\max _{\mathbf{x}, t}\left[\frac{1}{k} \lambda_{N, T}^{2}+k u_{N, T}^{2}\right]
\end{aligned}
$$

where $k$ is the search direction parameter. Note that subscripts $N$ and $T$ refer respectively to normal and tangential components at a given node belonging to the contacting interface. Criterion (25) is a very tough convergence indicator 


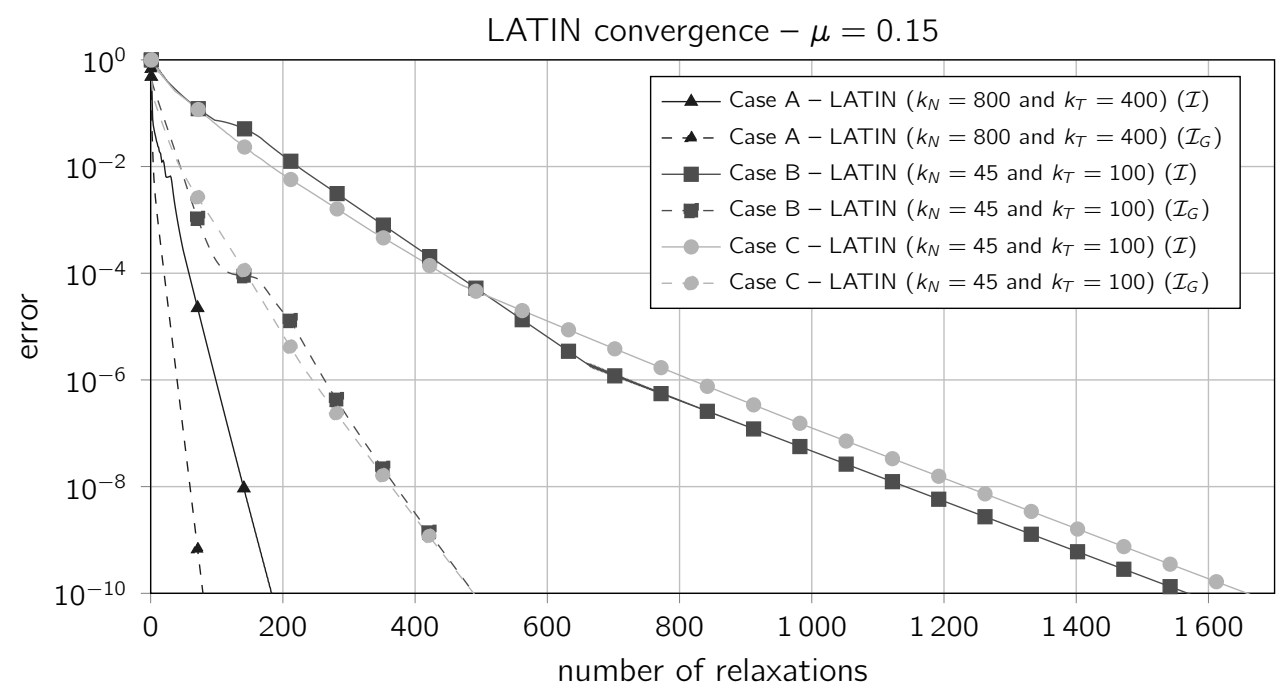

Figure 13: Convergence rates according to the local convergence criterion $\mathcal{I}$ and the global one $\mathcal{I}_{G}$ for cases A, B and C.

providing a possible low convergence rate. Another far less restrictive criterion (a global energetic one) can be used:

$$
\mathcal{I}_{\mathrm{G}}=\frac{\int_{0}^{T}\left(\mathbf{w}(t)-\mathbf{w}_{\mathrm{ref}}(t)\right)^{T} \mathbf{K}\left(\mathbf{w}(t)-\mathbf{w}_{\mathrm{ref}}(t)\right) \mathrm{d} t}{\int_{0}^{T} \mathbf{w}_{\mathrm{ref}}(t)^{T} \mathbf{K} \mathbf{w}_{\mathrm{ref}}(t) \mathrm{d} t}
$$

where $\mathbf{w}_{\text {ref }}$ is the reference solution for displacement field. As it proceeds in a global space-time approach implying a specific error appreciation (e.g. convergence criterion, constitutive law error...), LATIN method cannot be directly compared to usual methods.

The LATIN method is summarized up in the algorithm 1 and convergence indicators are plotted for all described cases on figure 13.

\subsection{Algorithm proposal}

The full approximation scheme (FAS) multigrid method [37] is a well-suited solver for non-linear problems. Difficulty of a such a solver is to define a coarse problem where low-cost corrections (computationally speaking) are calculated. For that purpose, we propose the following algorithm:

1. a few iterations ( $\nu_{1}$ relaxations) on the full order model (FOM) with the non-linear LATIN solver;

2. compute a coarse correction on a subspace spanned by space vectors (on a reduced order model or ROM);

3. a few iterations ( $\nu_{2}$ relaxations) on the full order model with the non-linear LATIN solver. 


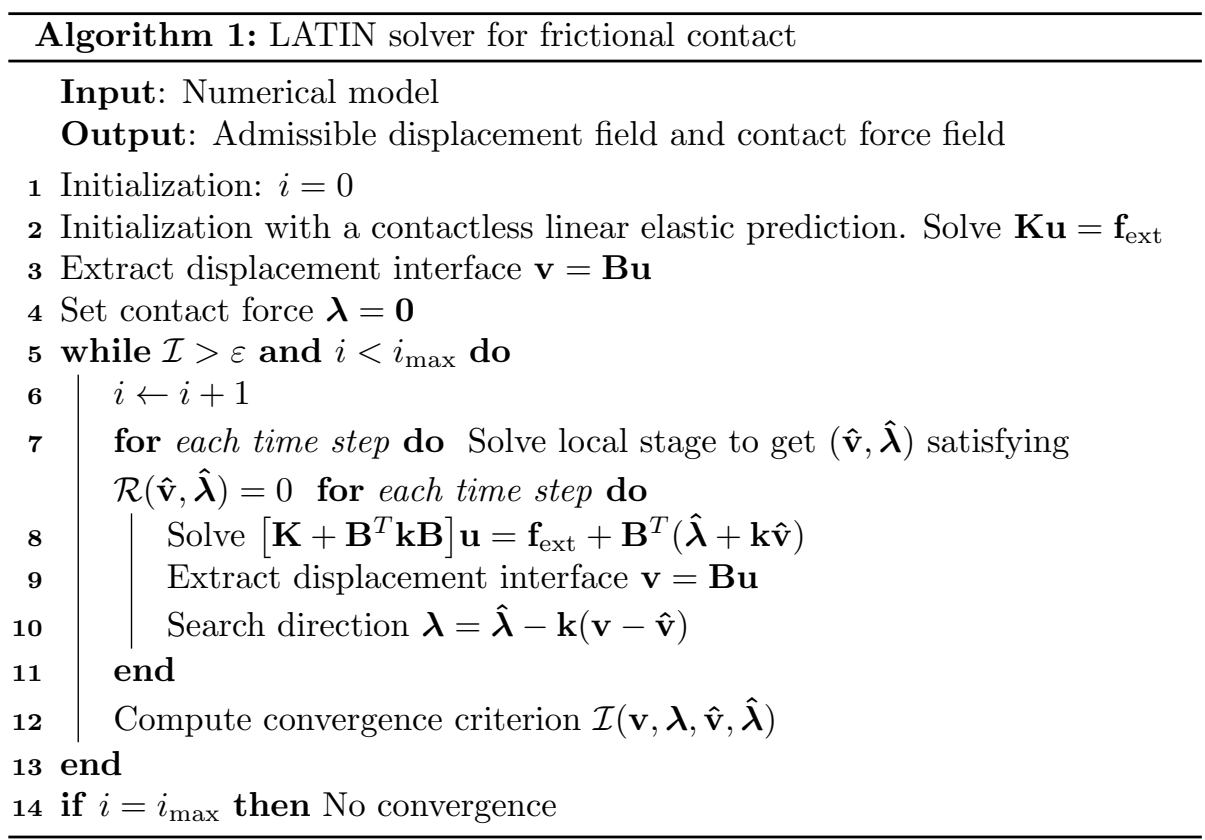

We have to define operators to transfer quantities between the full order space and the reduced order one. These are analog to intergrid operators (restriction and interpolation operators). Thus, we define:

- restriction operator projecting dual quantities from FOM to ROM: R (e.g. $\mathbf{R}=\boldsymbol{\Upsilon}^{T}$ )

- restriction operator projecting primal quantities from FOM to ROM: $\overline{\mathbf{R}}$ (e.g. $\overline{\mathbf{R}}=\boldsymbol{\Upsilon}^{T}$ )

- prolongation operator projection primal quantities from ROM to FOM: $\mathbf{P}=\boldsymbol{\Upsilon}($ e.g. $\mathbf{P}=\mathbf{\Upsilon})$

As mentioned in [39], prolongation and restriction operators have to fulfill energy conservation condition between ROM and FOM. Consequently, we have to verify :

$$
\mathbf{R}=\mathbf{P}^{T}
$$

Fine problem (FOM). LATIN iterations are considered as relaxations on the FOM (or the finest description of the problem). The solution of the FOM is the solution of the reference problem. Consequently, convergence indicator and errors have to be evaluated at this stage. We chose to end relaxations by a local stage that produces a solution $(\widetilde{\mathbf{u}}, \widetilde{\boldsymbol{\lambda}})$ satisfying the contact behavior but not admissibility conditions. There is therefore a balance residual defined as:

$$
\widetilde{\mathbf{r e s}}=\mathbf{f}_{\mathrm{ext}}+\mathbf{B}^{T} \widetilde{\boldsymbol{\lambda}}-\mathbf{K} \widetilde{\mathbf{u}}
$$

Coarse problem (ROM). We have to define it in such a way that we get relevant corrections for both displacement field and contact force field $(\widetilde{\mathbf{u}}, \widetilde{\boldsymbol{\lambda}})$ 
obtained from previous relaxation stage. For this purpose, we propose a mixed version of the LATIN solver and FAS coarse solver: a two-stage iterative process on corrective increments to satisfy contact conditions and reducing equilibrium residual.

- First stage. If one gets a first approximation of the correction $(\widehat{\Delta \mathbf{u}}, \widehat{\Delta \boldsymbol{\lambda}})$, we search for a better approximation $(\Delta \mathbf{u}, \Delta \boldsymbol{\lambda})$ trying to reduce the balance residual res which is:

$$
\operatorname{res}=\mathbf{f}_{\mathrm{ext}}+\mathbf{B}^{T}(\widetilde{\boldsymbol{\lambda}}+\Delta \boldsymbol{\lambda})-\mathbf{K}(\widetilde{\mathbf{u}}+\Delta \mathbf{u})
$$

using an additional search direction:

$$
\Delta \boldsymbol{\lambda}=\widehat{\Delta \boldsymbol{\lambda}}-\mathbf{k}(\Delta \mathbf{v}-\widehat{\Delta \mathbf{v}})
$$

one gets

$$
\mathbf{r e s}=\widetilde{\operatorname{res}}+\mathbf{B}^{T}(\widehat{\Delta \lambda}+\mathbf{k} \widehat{\Delta v})-\left[\mathbf{K}+\mathbf{B}^{T} \mathbf{k B}\right] \Delta \mathbf{u}
$$

The FAS scheme nullifies the coarse component of this residual (i.e. $\mathbf{R r e s}=$ 0) with the representation:

$$
\Delta \mathbf{u}=\mathbf{P}(\boldsymbol{\Psi}-\overline{\mathbf{R}} \widetilde{\mathbf{u}})
$$

Therefore it leads to solve:

$$
\begin{gathered}
{\left[\mathbf{R}\left[\mathbf{K}+\mathbf{B}^{T} \mathbf{k B}\right] \mathbf{P}\right](\mathbf{\Psi}-\overline{\mathbf{R}} \widetilde{\mathbf{u}})=\mathbf{R}\left[\widetilde{\operatorname{res}}+\mathbf{B}^{T}(\widehat{\Delta \boldsymbol{\lambda}}+\mathbf{k} \widehat{\Delta \mathbf{v}})\right]} \\
\Leftrightarrow\left[\mathbf{R}\left[\mathbf{K}+\mathbf{B}^{T} \mathbf{k B}\right] \mathbf{P}\right] \mathbf{\Psi}=\mathbf{R}\left[\widetilde{\mathbf{r e s}}+\mathbf{B}^{T}(\widehat{\Delta \boldsymbol{\lambda}}+\mathbf{k} \widehat{\Delta \mathbf{v}})+\left[\mathbf{K}+\mathbf{B}^{T} \mathbf{k B}\right] \mathbf{P} \overline{\mathbf{R}} \widetilde{\mathbf{u}}\right]
\end{gathered}
$$

Once $\boldsymbol{\Psi}$ is obtained, the correction $(\Delta \mathbf{u}, \Delta \boldsymbol{\lambda})$ is easily computed and the corresponding full fields are $(\widetilde{\mathbf{u}}+\Delta \mathbf{u}, \widetilde{\boldsymbol{\lambda}}+\Delta \boldsymbol{\lambda})$.

- Second stage. It consists in obtaining a new correction $(\widehat{\Delta \mathbf{u}}, \widehat{\Delta \boldsymbol{\lambda}})$ to satisfy contact conditions for the full fields. Here, we simply reuse the local stage to get full fields $(\widehat{\mathbf{u}}, \widehat{\boldsymbol{\lambda}})$ and corrective increments $(\widehat{\Delta \mathbf{u}}, \widehat{\Delta \boldsymbol{\lambda}})$.

The pseudocode is described in algorithm 2.

\subsection{Application}

Knowing a posteriori a reduced basis defining the coarse problem, we compare the proposed strategy and the former non-linear LATIN solver in terms of number of relaxations for each three problems presented before. Sets of parameters used for the application of the proposed algorithm are described on table 3 . The value of search direction parameters are set to optimal values (the less number of LATIN iterations are needed to solve the problem). 


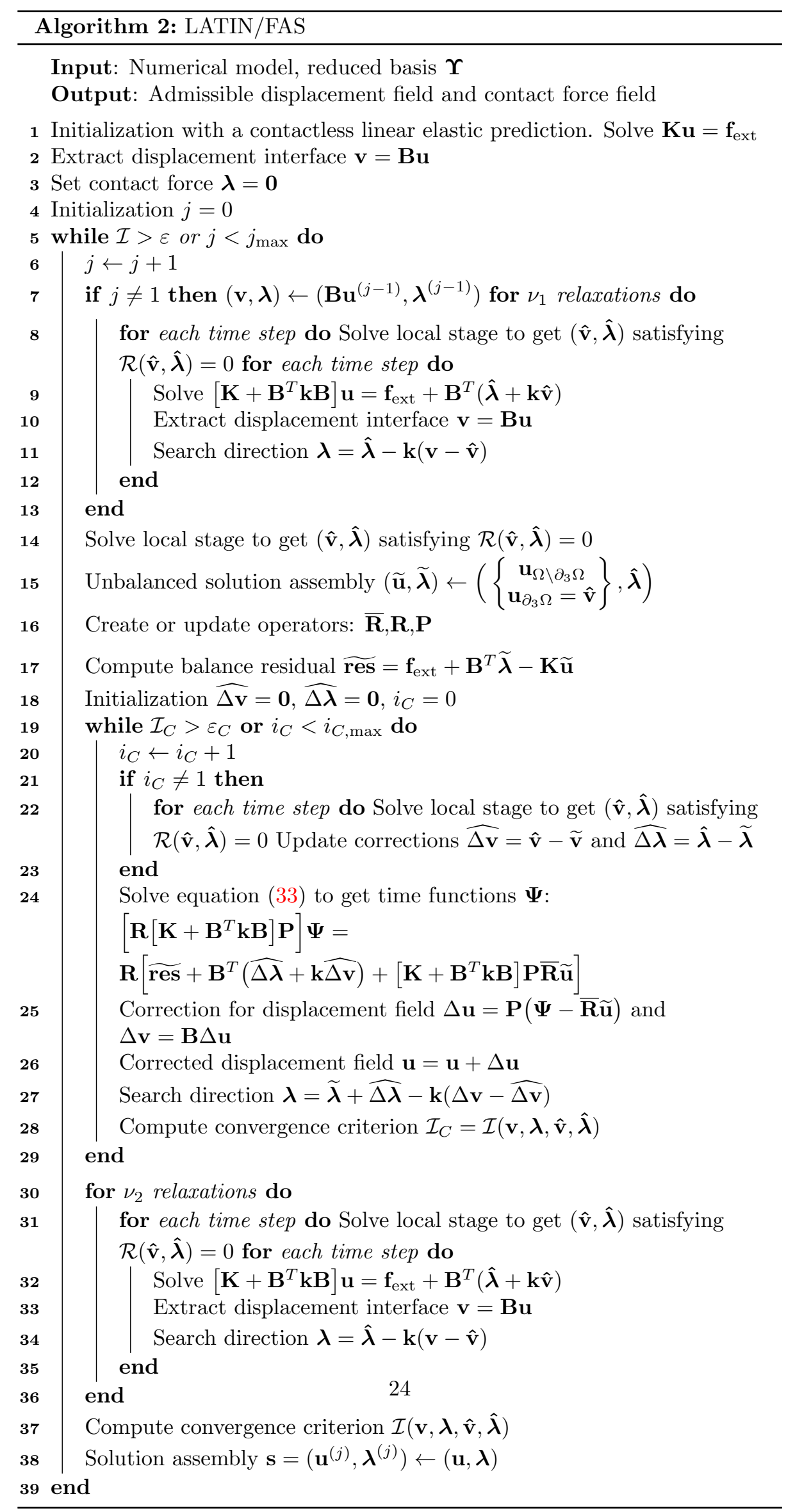




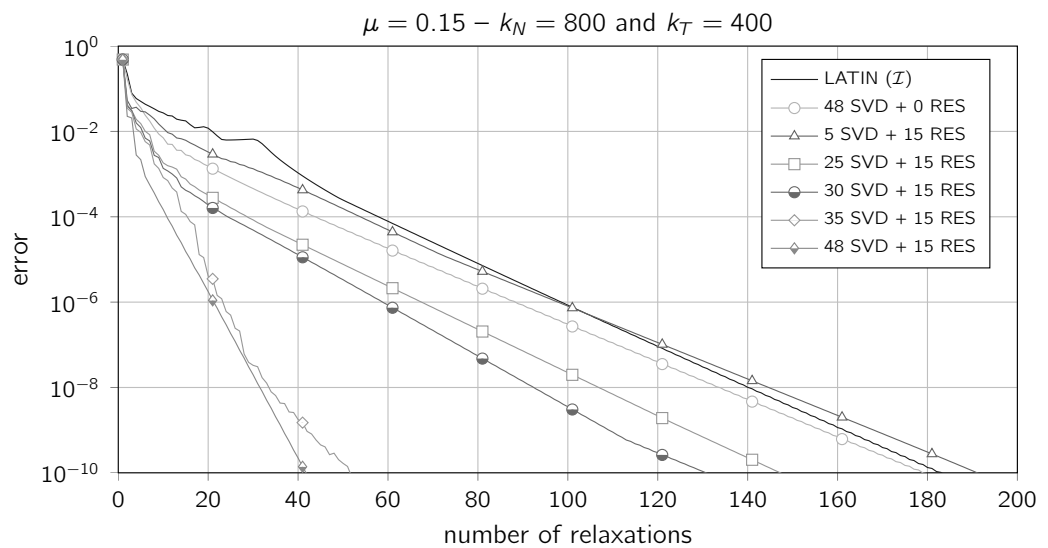

(a)

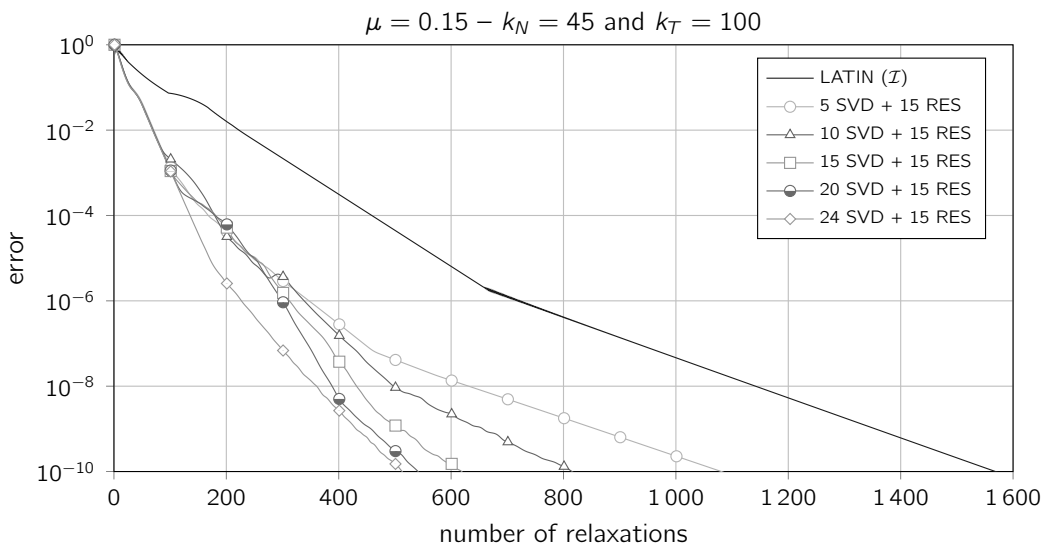

(b)

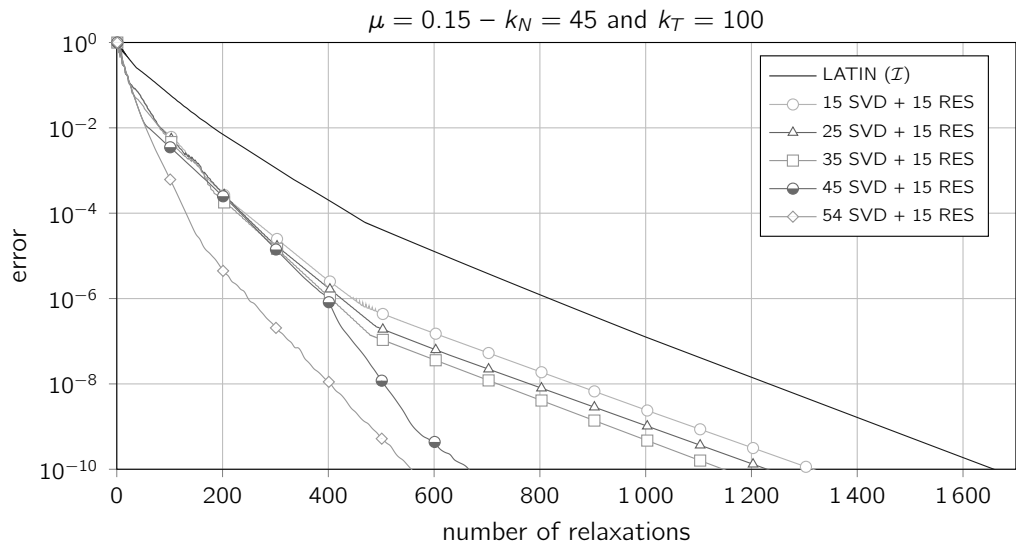

(c)

Figure 14: Convergence rates of the algorithm proposed vs. LATIN method: (a) Case A - (b) Case B - (c) Case C. Convergence diagrams are plotted with errors evaluated at each relaxation (coarse problem is considered costless). As no analytic solution exists, the reference is a solution evaluated with a precision of $\varepsilon=10^{-12}$. The number of SVD vectors defining the reduced order model is studied. 


\begin{tabular}{|c|c|c|c|}
\hline Parameters & Case A & Case B & Case C \\
\hline$\mu$ & \multicolumn{3}{|c|}{0.15} \\
\hline$\nu_{1}$ & 1 & 1 & 3 \\
\hline$\nu_{2}$ & 1 & 1 & 3 \\
\hline$i_{C, \max }$ & 15 & 15 & 15 \\
\hline$\varepsilon_{C}$ & -1 & -1 & -1 \\
\hline
\end{tabular}

Table 3: Algorithm parameters used for previous different cases.

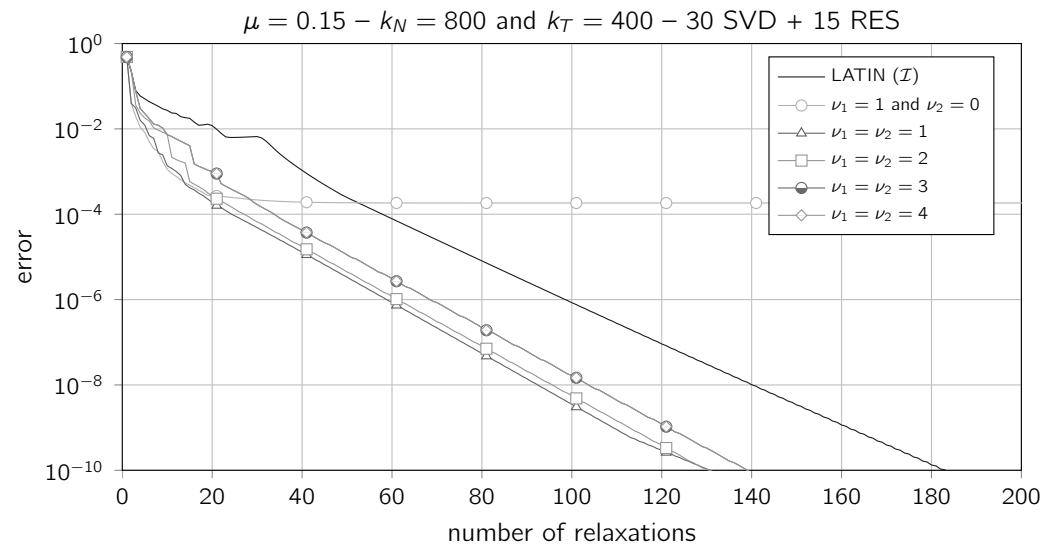

Figure 15: Convergence rates of the algorithm proposed vs. LATIN method for case A. Convergence diagrams are plotted with errors evaluated at each relaxation (coarse problem is considered costless). The number of relaxations before $\left(\nu_{1}\right)$ and after $\left(\nu_{2}\right)$ coarse stage is studied. 


\subsection{Discussion}

Results on figure 14 were obtained by enforcing coarse step iterating 15 times for each cycle (one cycle $=\nu_{1}$ relaxations + coarse step $+\nu_{2}$ relaxations). The threshold $\varepsilon_{C}$ is therefore set to a negative value to prescribe a given number of iterations for the coarse correction. In this study, we chose a rather large number of iterations for the coarse step to get an "exact" coarse correction. Indeed, it would be possible to make it only run until a threshold fixed by a level of accuracy (convergence criterion $\varepsilon_{C, \max }>0$ ).

Generally speaking, the proposed algorithm converges faster than the LATIN method (number of relaxations can be divided from 2 to 10 depending on parameters and desired precision).

On figure 14, the first comment concerns the content of the reduced basis and the definition of operators $\mathbf{P}, \mathbf{R}, \overline{\mathbf{R}}$. A reduced order model built exclusively on a posteriori SVD vectors leads to a weak acceleration of convergence even if the size of the basis is large (for case A, the 48 SVD vectors correspond to all vectors associated to non-null singular values). As a consequence, other vectors have to be introduced to define the coarse problem. Indeed, at each cycle, an approximated solution is built which could be not properly represented in the ROM (SVD vectors are built from the solution of the problem and not from the iterates). As a consequence, relevant coarse corrections can not be brought to the FOM from ROM. For that purpose, we have to add other vectors spanning the orthogonal residual (to SVD basis) of iterated solution. Arbitrary, we complete SVD vectors with 15 other vectors spanning the residual. At each cycle, these vectors are updated and the size of the coarse problem remains the same. On figure 14, we study the influence of the number of SVD vectors fixing the number of "residual vectors" to 15 .

Generally speaking, the more SVD vectors there are, the larger is the number of used SVD vectors, the smaeller is the number of iterations to converge, but the larger is the coarse problem. Consequently, we have to make a compromise between the size and the relevancy of the coarse model. A too few number of vectors defining the coarse problem makes the coarse step inefficient (no accelerating effect) or, even worse, tends to struggle convergence of the algorithm (coarse correction becomes irrelevant). The additional residual vectors allow to counterbalance a possible lake of relevancy of SVD vectors and ensure convergence.

FAS/LATIN convergence plots exhibit different regimes in the converging process: an acceleration of the convergence is clearly seen during a first group of iterations, then the convergence rate of the former classical LATIN method is recovered. The larger the reduced basis is, the longer the accelerating regime pertains. This can be explained by the multiscale content of the reduced basis as it was exemplified in section 3. During the first iterations, global scale of the problem is captured (and contained in the first modes of the SVD) involving a steep slope of the error. Then, the slope reduces depending on the content of the coarse problem. During this second regime, local scales are solved and higher order SVD vectors are used to compute coarse correction. The last regime shows a convergence rate roughly similar to the former LATIN method convergence rate. Only relaxation steps are useful and the coarse step does not bring corrections anymore.

Besides the definition of the coarse problem, other parameters can influence 
the behavior of the proposed algorithm, namely: the number of relaxations before and after the coarse step. This parameter allows to give a prominent role to the FOM or the ROM. Generally speaking, more relaxations steps renders the algorithm slower but more robust as shown on figure 15: a too few relaxations makes fail the convergence of the problem (up to a certain level of accuracy: for instance on figure $15, \nu_{1}=1$ and $\nu_{2}=0$ stops to converge at $10^{-4}$ ). This may also depend on the number of SVD vectors that are selected. According to results on figure 15, the less (but a sufficient) number of relaxations is the optimal configuration. Note that the more relaxations there are for $\nu_{1}$ and $\nu_{2}$, the closest to the LATIN method our strategy becomes (the less the coarse step occurs).

\section{Parametric study}

Product design requires several optimization phases. In particular, physical parameters has to be choose among discrete or continuous interval values in order to minimize a cost function. To achieve this goal, response surface methodology (using e.g. basic interpolations or kriging) can be used to identify the optimum set of parameters. A such methodology needs several evaluations of the cost function (a sample of set of parameters) which is a very expensive phase. Thus, acceleration strategies might be a very useful tool to save up computational cost. In this respect, we propose to deploy our strategy.

Originally, the LATIN method is very well-suited for such parametric studies. The main idea consists in re-using a previous solution for a given set of parameters to start LATIN iterations for a new set of parameters. This way, a large amount of relaxations (LATIN iterations on the full order model) can be spared as it is described in $[46,48,49]$.

In the other hand, reduced basis methods are also used to accelerate solutions [50, 51, 52]. Generally speaking, these consist in solving once the full-order problem associated to a first set of parameters and extract a reduced basis from computed solution (an expensive first phase). Then, the reduced basis is used (and eventually enriched) to accelerate solutions of other sets of parameters.

We wish study different values for the friction coefficient $\mu \in\left\{\mu_{m}=0,0.01,0.5,0.7, \mu_{M}=\right.$ $1\}$ focusing on the case A, knowing a first result for $\mu=0.15$.

First, we propose a reduced basis approach using our strategy. Then, a combination between reusing previous solution to compute new ones (as proposed in $[46,48])$ and our strategy is tested. All these approaches are compared to a brute force method.

\subsection{Brute force method}

For each $\mu$, a calculation is carried out with the non-linear full-order LATIN method (figure 16).

\subsection{Reduced basis methods}

Two approaches can be used [53]:

(I) First ROM-POD approach. A reduced basis is built from the solution with $\mu=0.15$. Then, this basis is used to solve all other problems associated 
to the other friction coefficients.

(II) Second ROM-POD approach. A reduced basis is constructed from resolutions of problem associated to extreme values for the varying parameter $(\mu=0$ and $\mu=1)$. Then, we use this basis to solve all problems associated to all other $\mu$. SVD reduced basis is built by concatenating and orthogonalizing the first $20 \mathrm{SVD}$ vectors of the solution for $\mu=0$ and the first 20 SVD vectors of the solution for $\mu=1$.

For both ROM-POD approaches, we update a set of vectors of the reduced basis on the fly without a posteriori knowledge, but we keep the same size of the reduced basis.

Results obtained using the proposed algorithm are depicted on figure 16. Clearly, using our strategy to solve similar problems with varying parameters allows to reduce the number of relaxations in comparison to brute force method. Bringing coarse corrections on reduced basis (global scale of the structure) is relevant for all problems differing only on a localized scale (contacting boundary). Generally speaking, in both approaches, simulations with small coefficients of friction ( $\mu=0$ and $\mu=0.01)$ are highly accelerated. But for higher ones, accelerating effect is weaker for both approaches. That can be explained by the toughness of tangential problem which is difficult for large coefficient of friction requiring particular local modes. Moreover the SVD basis is built with a problem where the friction coefficient is relatively small $(\mu=0.15)$. Approach (II) is globally more efficient than approach (I). Indeed, coarse model of approach (II) is built from a larger panel of solutions and is spanned with more vectors.

Enriching the collection of SVD vectors with vectors spanning the unprojected residual adapts the definition the coarse stage to a specific problem to solve. It can be seen as an a priori method which tries to maintain the relevancy of the subspace spanned by the the coarse model.

\subsection{Reusing previous solutions}

In this paper, we initialize the LATIN and the FAS/LATIN method with the linear elastic solution (kinematically and statically admissible) without contact conditions. Now, as in $[46,48]$, we initialize the LATIN method or FAS/LATIN with the previous solution computed (figure 17). We compare the number of relaxations done to reach a precision of $10^{-4}$ for all approaches (figure 18). For the FAS/LATIN, the first reduced basis approach is used (basis computed for $\mu=0.15)$.

We firstly note that the solution reusing doesn't reduce the number of relation for LATIN method. Indeed, the linear elastic solution (used originally) of the studied case is already a good and relevant first guess of the low-frictional solutions for case A. In case of $\mu=0$ (frictionless problem), the solution for $\mu=0.15$ is irrelevant (mid-frictional problem) and the number of relaxations increases. Nevertheless, for high-frictional problems $(\mu=0.5$ or 0.7$)$, initializing with a (high-)frictional solution is this time a relevant choice. A quite obvious conclusion can be drawn, reusing solutions is suited for initializing LATIN to solve quite similar problems. In addition to this observation, the FAS/LATIN algorithm provides a very interesting gain and shows its ability to perform such parametric problems. 


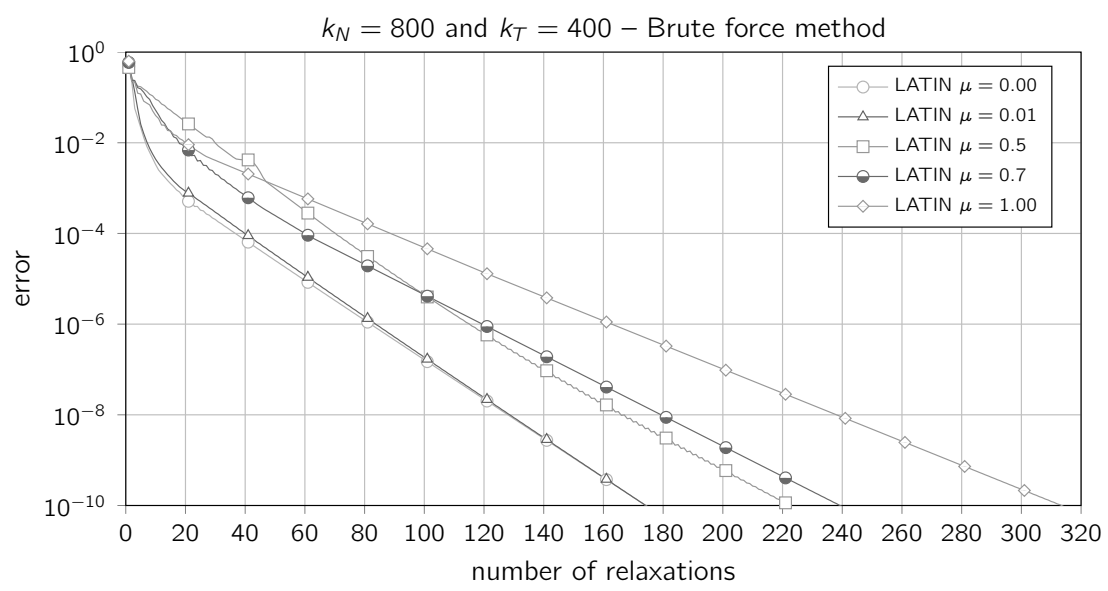

(a)

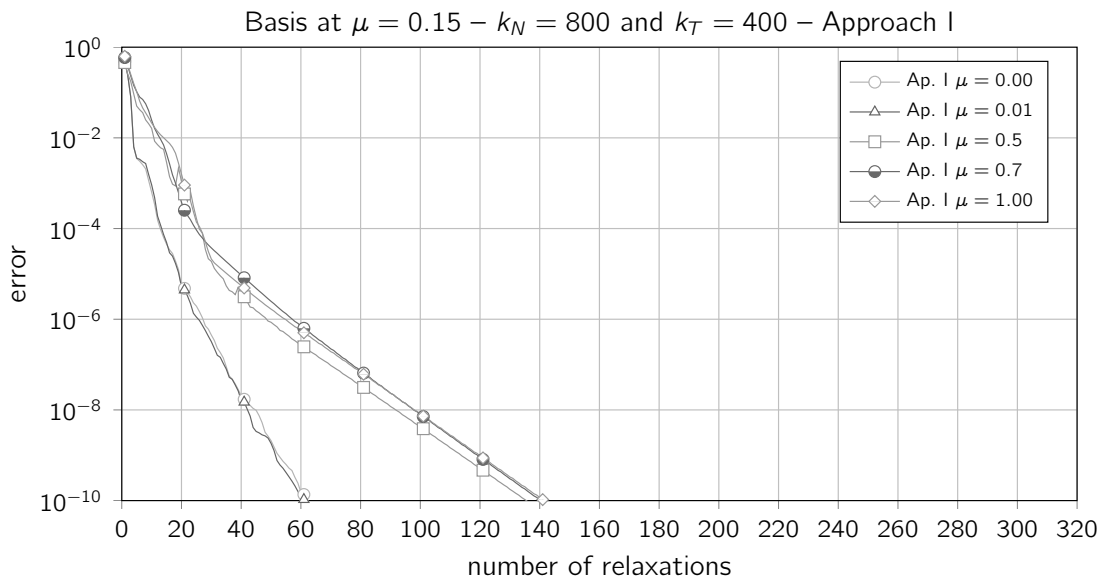

(b)

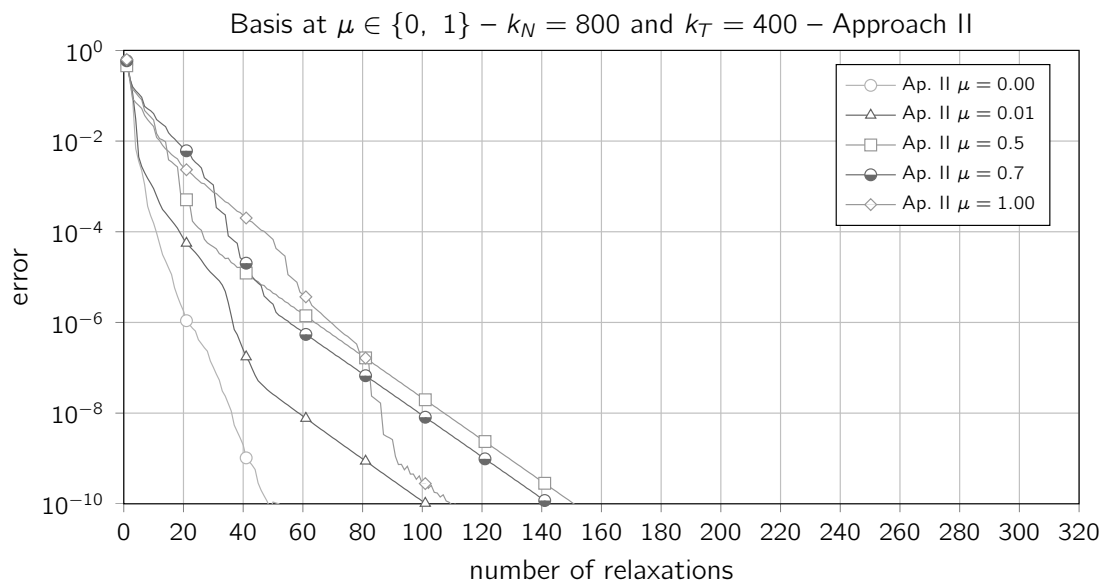

(c)

Figure 16: Convergence rate according different methods. (a) : Brute force method (LATIN method is used to solve each problem) - (b) : approach (I): $\nu_{1}=\nu_{2}=2, k_{C}=15, \varepsilon_{C}=-1,40$ modes from SVD of the solution $\mu=0.15$ +15 residual vectors. (c) : approach (39): $\nu_{1}=\nu_{2}=2, k_{C}=15, \varepsilon_{C}=-1$. 


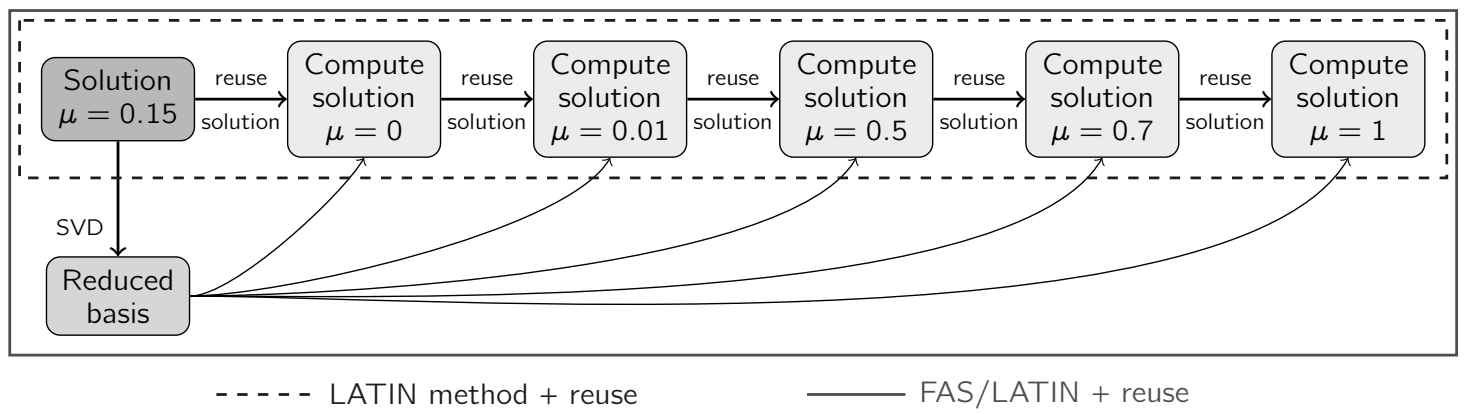

Figure 17: LATIN and FAS/LATIN using the previous solution computed.

Number of relaxations to reach $\mathcal{I}_{L}<10^{-4}$

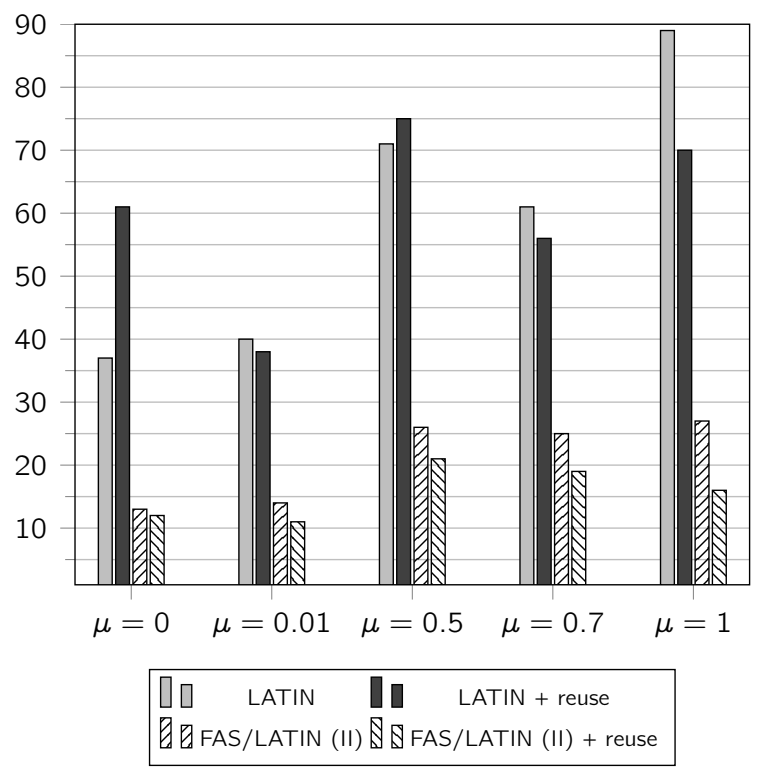

Figure 18: LATIN and FAS/LATIN using the previous solution computed. 


\section{Conclusion}

This article shows that a frictional contact problem can be reduced and efficiently described with a set of space-time radial functions (radial solutions) provided that a time-space model reduction is used. Analyses of each radial solution reveal a clear scale separability of the solution. This observation justifies the use of multiscale approach. Then, an algorithm based on the non-linear FAS multigrid solver was proposed. The relaxation step is based on the non-linear LATIN solver and the non-linear coarse step is based on a reduced basis strategy. However, a sole POD basis is insufficient to increase efficiently the convergence rate even if the solution of one problem is highly reducible. This is why a few more vectors have to complete the POD basis: the iterates of solutions built by the non-linear iterative solver have to be taken into account. Consequently, a possible enrichment of the reduced basis is a key point to increase efficiently the convergence rate of the multiscale approach. Moreover, it has been shown that such an approach is also well-suited to parametric studies.

In the proposed approach, enrichment of POD basis vectors with residual vectors can be compared with an a priori method. This enrichment can be integrated by considering the so-called radial approximation (or separated representation). The efficiency of this approach has already been emphasized for thermal and mechanical problems [54, 53, 44] which is close to PGD (Proper Generalized Decomposition) methods $[55,56]$. PGD methods operate without a priori knowledge of the solution (contrary to POD approaches). Many works underline the potential of such a priori approaches $[53,57]$. That point is currently being investigated.

Acknowledgement We gratefully acknowledge the French National Association for Research and Technology (ANRT, CIFRE grant number 2011/0705).

\section{References}

[1] Fortin M, Glowinski R. Augmented Lagrangian methods: applications to the numerical solution of boundary-value problems. Access Online via Elsevier, 2000.

[2] Simo J, Laursen T. An augmented lagrangian treatment of contact problems involving friction. Computers \& Structures 1992; 42(1):97-116.

[3] Laursen TA. Formulation and treatment of frictional contact problems using finite elements. PhD Thesis, Stanford University 1992.

[4] Wriggers P. Finite element algorithms for contact problems. Archives of Computational Methods in Engineering 1995; 2:1-49.

[5] Heinstein M, Laursen TA. An algorithm for the matrix-free solution of quasistatic frictional contact problems. International Journal for Numerical Methods in Engineering 1999; 44(9):1205-1226, doi:10.1002/(SICI) 1097-0207(19990330)44:9〈1205::AID-NME550〉3.0.CO;2-0.

[6] Alart P, Dureisseix D. A scalable multiscale latin method adapted to nonsmooth discrete media. Computer Methods in Applied Mechanics and Engineering 2008; 197(5):319-331. 
[7] Bussetta P, Marceau D, Ponthot JP. The adapted augmented lagrangian method: a new method for the resolution of the mechanical frictional contact problem. Computational Mechanics 2012; 49(2):259-275.

[8] Pietrzak G, Curnier A. Large deformation frictional contact mechanics: continuum formulation and augmented lagrangian treatment. Computer Methods in Applied Mechanics and Engineering 1999; 177(3):351-381.

[9] Jourdan F, Alart P, Jean M. A gauss-seidel like algorithm to solve frictional contact problems. Computer methods in applied mechanics and engineering 1998; 155(1):31-47.

[10] Jean M. The non-smooth contact dynamics method. Computer methods in applied mechanics and engineering 1999; 177(3):235-257.

[11] Dostál Z, Haslinger J, Kučera R. Implementation of the fixed point method in contact problems with coulomb friction based on a dual splitting type technique. Journal of computational and applied mathematics 2002; $140(1): 245-256$.

[12] Barboteu M, Alart P, Vidrascu M. A domain decomposition strategy for nonclassical frictional multi-contact problems. Computer Methods in Applied Mechanics and Engineering 2001; 190(37):4785-4803.

[13] Dureisseix D, Farhat C. A numerically scalable domain decomposition method for the solution of frictionless contact problems. International Journal for Numerical Methods in Engineering 2001; 50(12):2643-2666.

[14] Renouf M, Alart P. Conjugate gradient type algorithms for frictional multicontact problems: applications to granular materials. Computer Methods in Applied Mechanics and Engineering 2005; 194(18):2019-2041.

[15] Dostál Z, Schoberl J. Minimizing quadratic functions subject to bound constraints with the rate of convergence and finite termination. Computational Optimization and Applications 2005; 30(1):23-43.

[16] Curnier A, Alart P. A generalized newton method for contact problems with friction. Journal de mécanique théorique et appliquée 1988; 7:67-82.

[17] Alart P. Méthode de newton généralisée en mécanique du contact. Journal de mathématiques pures et appliquées 1997; 76(1):83-108.

[18] Li D, Fukushima M. Smoothing newton and quasi-newton methods for mixed complementarity problems. Computational Optimization and Applications 2000; $17(2-3): 203-230$.

[19] Renard Y. Generalized newtons methods for the approximation and resolution of frictional contact problems in elasticity. Computer Methods in Applied Mechanics and Engineering 2012; 256:38-55.

[20] Anitescu M, Potra FA. Formulating dynamic multi-rigid-body contact problems with friction as solvable linear complementarity problems. Nonlinear Dynamics 1997; 14(3):231-247. 
[21] Alart P, Lebon F. Solution of frictional contact problems using ilu and coarse/fine preconditioners. Computational mechanics 1995; 16(2):98-105.

[22] Lebon F, Raous M, Rosu I. Multigrid methods for unilateral contact problems with friction. IUTAM Symposium on Computational Methods in Contact Mechanics, IUTAM Bookseries, vol. 3. 2007; 1-16, doi:10.1007/ 978-1-4020-6405-0_1.

[23] Kerfriden P, Gosselet P, Adhikari S, Bordas S. Bridging proper orthogonal decomposition methods and augmented newton-krylov algorithms: An adaptive model order reduction for highly nonlinear mechanical problems. Computer Methods in Applied Mechanics and Engineering 2011; 200:850 - 866, doi:10.1016/j.cma.2010.10.009.

[24] Kunisch K, Volkwein S. Galerkin proper orthogonal decomposition methods for parabolic problems. Numerische Mathematik 2001; 90:117-148, doi: $10.1007 / \mathrm{s} 002110100282$.

[25] Ryckelynck D, Chinesta F, Cueto E, Ammar A. On the a priori model reduction: Overview and recent developments. Archives of Computational Methods in Engineering 2006; 13:91-128, doi:10.1007/BF02905932.

[26] Amsallem D, Zahr MJ, Farhat C. Nonlinear model order reduction based on local reduced-order bases. International Journal for Numerical Methods in Engineering 2012; 92(10):891-916, doi:10.1002/nme.4371.

[27] Ladevèze P. Nonlinear Computational Structural Methods: New Approaches and Non-Incremental Methods of Calculation. Springer-Verlag New York Inc., 1999.

[28] Champaney L, Cognard JY, Dureisseix D, Ladevèze P. Large scale applications on parallel computers of a mixed domain decomposition method. Computational Mechanics 1997; 19:253-263, doi:10.1007/s004660050174.

[29] Galland F, Gravouil A, Malvesin E, Rochette M. A global model reduction approach for 3D fatigue crack growth with confined plasticity. Computer Methods in Applied Mechanics and Engineering 2011; 200:699 - 716, doi: 10.1016/j.cma.2010.08.018.

[30] Galland F. An adaptive model reduction approach for 3D fatigue crack growth in small scale yielding conditions. PhD Thesis, INSA de Lyon 2011.

[31] Signorini A. Questioni di elasticità non linearizzata e semilinearizzata. Rendiconti di Matematica e delle sue applicazioni, V. Ser. 1959; 18:95-139.

[32] Coulomb CA. Théorie des machines simples. Mémoires de l'Académie des Sciences, 1785 .

[33] Eckart C, Young G. The approximation of one matrix by another of lower rank. Psychometrika 1936; 1:211-218.

[34] Brandt A. Multiscale solvers and systematic upscaling in computational physics. Computer Physics Communications 2005; 169:438 - 441, doi:10. 1016/j.cpc.2005.03.097. 
[35] Pierres E, Baietto MC, Gravouil A. A two-scale extended finite element method for modelling 3D crack growth with interfacial contact. Computer Methods in Applied Mechanics and Engineering 2010; 199:1165 - 1177, doi:10.1016/j.cma.2009.12.006.

[36] Kerfriden P, Passieux J, Bordas S. Local/global model order reduction strategy for the simulation of quasi-brittle fracture. International Journal for Numerical methods in Engineering 2011; 89:154-179.

[37] Venner CH, Lubrecht AA. Multilevel Methods in Lubrication. Elsevier, 2000.

[38] Cavin P, Gravouil A, Lubrecht AA, Combescure A. Efficient FEM calculation with predefined precision through automatic grid refinement. Finite Elements in Analysis and Design 2005; 41:1043-1055, doi:10.1016/j.finel. 2004.09.004.

[39] Rannou J. Prise en compte d'effets d'échelle en mécanique de la rupture tridimensionnelle par une approche X-FEM multigrille localisée nonlinéaire. PhD Thesis, INSA de Lyon 2008.

[40] Biotteau E, Gravouil A, Lubrecht AA, Combescure A. Three dimensional automatic refinement method for transient small strain elastoplastic finite element computations. Computational Mechanics 2012; 49:123 - 136, doi: 10.1007/s00466-011-0628-z.

[41] Champaney L. Une nouvelle approche modulaire pour l'analyse d'assemblages de structures tridimensionnelles. PhD Thesis, ENS de Cachan 1996.

[42] Champaney L, Cognard JY, Ladevèze P. Modular analysis of assemblages of three-dimensional structures with unilateral contact conditions. Computers and Structures 1999; 73:249-266.

[43] Ladevèze $\mathrm{P}$, Lemoussu $\mathrm{H}$, Boucard $\mathrm{P}$. A modular approach to 3-D impact computation with frictional contact. Computers and Structures 2000; 78:45 - 51, doi:10.1016/S0045-7949(00)00094-8.

[44] Lemoussu H, Boucard PA, Ladevèze P. A 3D shock computational strategy for real assembly and shock attenuator. Advances in Engineering Software 2002; 33:517 - 526, doi:10.1016/S0965-9978(02)00074-1.

[45] Trollé B, Gravouil A, Baietto MC, Nguyen-Tajan T. Optimization of a stabilized X-FEM formulation for frictional cracks. Finite Elements in Analysis and Design 2012; 59:18 - 27, doi:10.1016/j.finel.2012.04.010.

[46] Boucard PA, Champaney L. A suitable computational strategy for the parametric analysis of problems with multiple contact. International Journal for Numerical Methods in Engineering 2003; 57(9):1259-1281.

[47] Ribeaucourt R, Baietto-Dubourg MC, Gravouil A. A new fatigue frictional contact crack propagation model with the coupled X-FEM/LATIN method. Computer Methods in Applied Mechanics and Engineering 2007; 196:3230 - 3247, doi:10.1016/j.cma.2007.03.004. 
[48] Odièvre D, Boucard PA, Gatuingt F. A parallel, multiscale domain decomposition method for the transient dynamic analysis of assemblies with friction. Computer Methods in Applied Mechanics and Engineering 2010; 199(21):1297-1306.

[49] Soulier B, Boucard PA. A multiparametric strategy for the two step optimization of structural assemblies. Structural and Multidisciplinary Optimization $2013 ;$ 47(4):539-553.

[50] Bolzon G, Buljak V. An effective computational tool for parametric studies and identification problems in materials mechanics. Computational Mechanics $2011 ; 48(6): 675-687$.

[51] Deparis S, Rozza G. Reduced basis method for multi-parameter-dependent steady navier-stokes equations: Applications to natural convection in a cavity. Journal of Computational Physics 2009; 228(12):4359-4378.

[52] Niroomandi S, Alfaro I, Cueto E, Chinesta F. Real-time deformable models of non-linear tissues by model reduction techniques. Computer Methods and Programs in Biomedicine 2008; 91(3):223-231.

[53] Heyberger C, Boucard PA, Néron D. Multiparametric analysis within the proper generalized decomposition framework. Computational Mechanics 2012; 49:277-289, doi:10.1007/s00466-011-0646-x.

[54] Ladevèze P, Passieux JC, Néron D. The LATIN multiscale computational method and the proper generalized decomposition. Computer Methods in Applied Mechanics and Engineering 2010; 199:1287 - 1296, doi:10.1016/j. cma.2009.06.023.

[55] Chinesta F, Ammar A, Cueto E. Recent advances and new challenges in the use of the proper generalized decomposition for solving multidimensional models. Archives of Computational Methods in Engineering 2010; 17:327350, doi:10.1007/s11831-010-9049-y.

[56] Chinesta F, Ladevèze $\mathrm{P}$, Cueto E. A short review on model order reduction based on proper generalized decomposition. Archives of Computational Methods in Engineering 2011; 18:395-404, doi:10.1007/s11831-011-9064-7.

[57] Boucinha L, Gravouil A, Ammar A. Space-time proper generalized decompositions for the resolution of transient elastodynamic models. Computer Methods in Applied Mechanics and Engineering 2013; 255:67 - 88, doi: 10.1016/j.cma.2012.11.003. 